\title{
Rheology of discrete failure regimes of anisotropic sea ice
}

Article

Published Version

Wilchinsky, A. V. and Feltham, D. L. (2012) Rheology of discrete failure regimes of anisotropic sea ice. Journal of Physical Oceanography, 42 (7). pp. 1065-1082. ISSN 15200485 doi: https://doi.org/10.1175/JPO-D-11-0178.1 Available at https://centaur.reading.ac.uk/34580/

It is advisable to refer to the publisher's version if you intend to cite from the work. See Guidance on citing.

To link to this article DOI: http://dx.doi.org/10.1175/JPO-D-11-0178.1

Publisher: American Meteorological Society

All outputs in CentAUR are protected by Intellectual Property Rights law, including copyright law. Copyright and IPR is retained by the creators or other copyright holders. Terms and conditions for use of this material are defined in the End User Agreement.

\section{www.reading.ac.uk/centaur}

\section{CentAUR}

Central Archive at the University of Reading

Reading's research outputs online 


\title{
Rheology of Discrete Failure Regimes of Anisotropic Sea Ice
}

\author{
AleXANDer V. Wilchinsky And Daniel L. Feltham \\ National Centre for Earth Observation: Centre for Polar Observation and Modelling, University College London, \\ London, United Kingdom
}

(Manuscript received 28 September 2011, in final form 19 December 2011)

\begin{abstract}
A rheological model of sea ice is presented that incorporates the orientational distribution of ice thickness in leads embedded in isotropic floe ice. Sea ice internal stress is determined by coulombic, ridging and tensile failure at orientations where corresponding failure criteria are satisfied at minimum stresses. Because sea ice traction increases in thinner leads and cohesion is finite, such failure line angles are determined by the orientational distribution of sea ice thickness relative to the imposed stresses. In contrast to the isotropic case, sea ice thickness anisotropy results in these failure lines becoming dependent on the stress magnitude. Although generally a given failure criteria type can be satisfied at many directions, only two at most are considered. The strain rate is determined by shearing along slip lines accompanied by dilatancy and closing or opening across orientations affected by ridging or tensile failure. The rheology is illustrated by a yield curve determined by combining coulombic and ridging failure for the case of two pairs of isotropically formed leads of different thicknesses rotated with regard to each other, which models two events of coulombic failure followed by dilatancy and refreezing. The yield curve consists of linear segments describing coulombic and ridging yield as failure switches from one lead to another as the stress grows. Because sliding along slip lines is accompanied by dilatancy, at typical Arctic sea ice deformation rates a one-day-long deformation event produces enough open water that these freshly formed slip lines are preferential places of ridging failure.
\end{abstract}

\section{Introduction}

Sea ice forms from the freezing of polar waters and covers a significant fraction, up to almost $10 \%$, of the earth's oceans. Sea ice is well recognized as an important component of the earth's climate system and, as a result, sea ice models are routinely incorporated into global climate models (GCMs). Although sea ice affects polar and global climate through its impact on the thermohaline budget (e.g., through its high albedo compared to seawater), its insulating effect on polar oceans, and its contribution to the freshwater balance when it melts or freezes, the focus of this paper is on sea ice dynamics or, more particularly, sea ice rheology. Sea ice dynamics is the catch-all name given to the combination of processes that move and deform the sea ice cover, which is described in GCMs by a combination of a momentum balance equation, mass balance equation, and a constitutive

Corresponding author address: Alexander Wilchinsky, NCEO: CPOM, Department of Earth Sciences, University College London, London WC1E 6BT, United Kingdom.

E-mail: aw@cpom.ucl.ac.uk law relating internal sea ice stresses to the sea ice state and deformation (sea ice rheology).

Much of the sea ice cover is comprised of brittle floes that are of approximately convex, polygonal shape with lateral dimensions ranging between about $100 \mathrm{~m}$ and $5 \mathrm{~km}$ and with thicknesses of several meters: in winter, the floes are typically frozen together to form a moreor-less continuous, heterogeneous cover of the ocean, whereas in summer the floes separate and a more dilute ice cover is typical. The modes of sea ice deformation that are considered important for geophysical-scale (e.g., $5 \mathrm{~km}$ and greater) sea ice models, which are considered in this paper, are pressure ridging, coulombic shear rupture and subsequent sliding, and tensile opening. Pressure ridging occurs when, under sufficient compressive stresses, the ice floes break up and override to form long piles of rubble above and beneath the ice cover (the pressure ridges and associated keels). Coulombic shear rupture occurs when shear stresses, under moderate confinement, cause the ice cover to rupture along pairs of lines traversing the ice pack. Because shear rupture is typically followed by frictional sliding along these lines, the lines are frequently known as slip or 
sliding lines. Tensile opening, as the name suggests, is caused by tensile (opening) stresses that break the ice in tension. This paper relates sea ice stresses to the mode and orientation of sea ice deformation more explicitly than previous treatments of sea ice rheology.

Since the Arctic Ice Dynamics Joint Experiment (AIDJEX) (Coon et al. 1974), sea ice rheology has been described as plastic, often by analogy of sea ice to granular materials. With few exceptions, these rheology models have treated the ice cover as isotropic: an assumption that rests upon the premise that the subcontinuum sea ice state (described by, e.g., the number, orientation, and thicknesses of leads) is isotropically distributed and/or the underlying material rheology of sea ice is intrinsically isotropic. Note that in practical applications the continuum scale is defined by the gridcell size used in the numerical sea ice model and the time step of the numerical model; at the time of the AIDJEX study, such scales were on the order of $100 \mathrm{~km}$ and 1 day. Another frequently used assumption is that geophysicalscale sea ice has no tensile strength. The assumption of isotropy has allowed classical plastic flow theory to be applied to sea ice, with the main distinguishing feature between alternate-competing sea ice rheology models being the shape of the plastic yield curve, the direction of the plastic flow law, and the nature of the subplastic yield rheology (viscous or elastic). The developed yield curve envelopes included the most used elliptic (Hibler 1979), sine-wave (Bratchie 1984), ice cream cone (Shen et al. 1987; Pritchard 1988; Hopkins 1996; Hibler and Schulson 2000), and linear coulombic shapes (Marko and Thomson 1977; Smith 1983; Overland and Pease 1988; Tremblay and Mysak 1997). See Feltham (2008) for a review of developments in modeling of sea ice rheology.

Satellite imagery (e.g., Fig. 1) shows that, in the winter pack, floes are frozen together and the sea ice cover fails discretely along linear failure lines at acute angles (Marko and Thomson 1977; Erlingsson 1988; Pritchard 1988), which are observed at a range of scales (e.g., 10$150 \mathrm{~km}$; Walter et al. 1995). Such imagery has lent weight to theories of discrete, coulombic fracture of the ice cover (e.g., Marko and Thomson 1977; Schulson 2001), with the internal friction (shear rupture) coefficient being independent of scale (Weiss and Schulson 2009). Moreover, sea ice stress measurements by RichterMenge et al. (2002) during the Surface Heat Budget of the Arctic Ocean (SHEBA) experiment (Perovich et al. 1999) recorded tensile stresses, usually ignored in sea ice models, as large as compressive stresses, which implies a significant cohesion if sea ice is assumed to fail coulombically (Weiss et al. 2007). The new data, as well as understanding of the sea ice processes, has debunked the earlier AIDJEX assumptions of isotropy and zero tensile
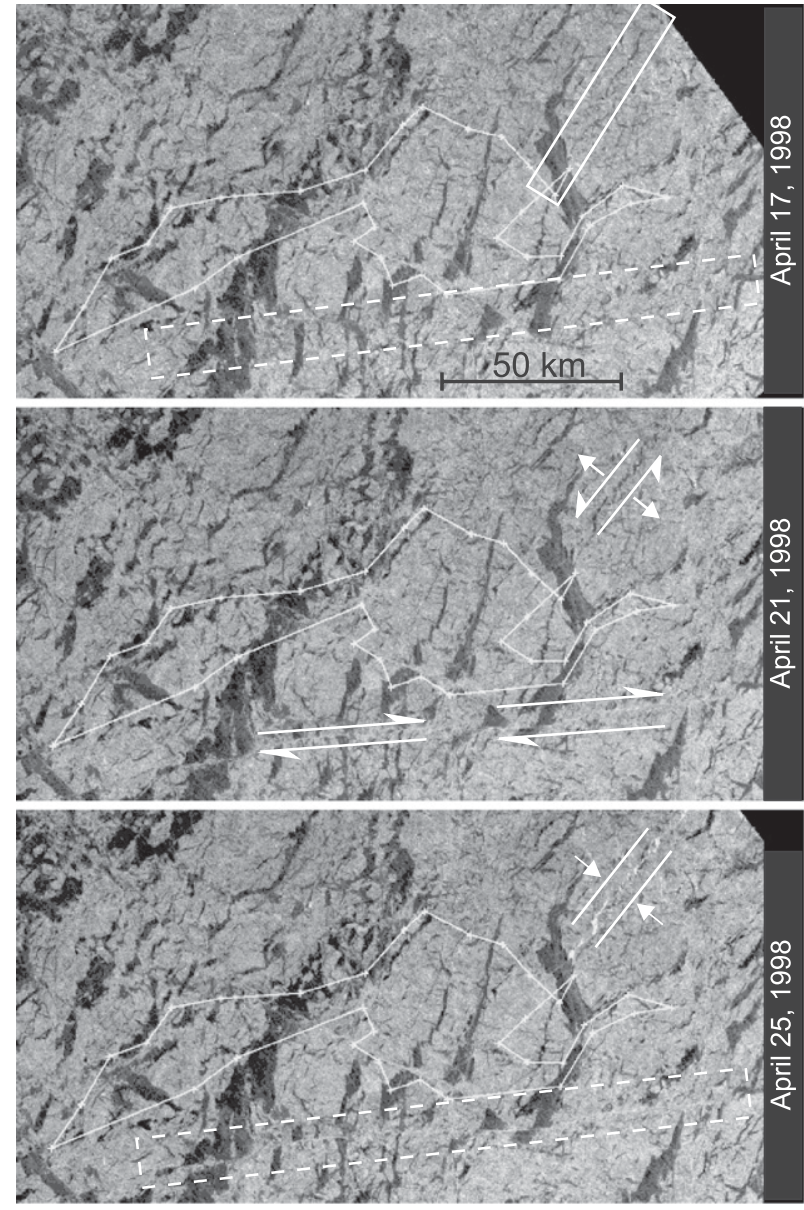

FIG. 1. An example of coulombic and ridging failure of sea ice during the SHEBA experiment in the Beaufort Sea as seen from synthetic aperture radar (SAR) images. The dates are given on the black margins. Darker areas show thinner ice. The white broken line comes from the original image sequence and delineates a region that was originally a square but has eventually been deformed into this shape. (top) The white rectangles (17 Apr) show positions of an existing lead (solid line) and a future lead (dashed line) probably formed through coulombic failure. (middle) By 21 Apr, the existing lead shears and opens due to dilatancy, and the new lead is formed through shear but with no visible dilatancy. (bottom) By $25 \mathrm{Apr}$, the old lead ridges as is shown by the appeared white color, whereas the new lead, shown again by a dashed rectangle, remains inactive. The images are provided by R. Kwok (2011, Jet Propulsion Laboratory, personal communication) [Radarsat-1 images from Canadian Space Agency (CSA)] and are available online (http://www-radar.jpl.nasa.gov/rgps/image_files/combine_small.gif).

strength (e.g., Coon et al. 2007) in favor of models that can describe discontinuity of the sea ice deformation (e.g., Hopkins and Thorndike 2006; Schreyer et al. 2006) and induced anisotropy due to highly aligned leads that may extend across large areas of the ice pack.

The effect of anisotropy has been treated either explicitly through consideration of particular leads (e.g., Coon et al. 1998; Hibler and Schulson 2000; Hibler 2001; 
Schreyer et al. 2006; Wilchinsky and Feltham 2011) or implicitly through continuum representation of anisotropy using heuristic arguments (Wilchinsky and Feltham 2004, 2006a). Additionally, Wilchinsky and Feltham (2006b), motivated by satellite imagery, treated the ice cover as comprising diamond-shaped ice blocks formed from intersecting slip lines, to develop an anisotropic sea ice model avoiding detailed modeling of fracture processes. Because of its simplicity this latter model allowed a straightforward incorporation into the sea ice component of a climate model (Tsamados et al. 2012, manuscript submitted to J. Geophys. Res.). Although the necessity of representing of the discrete features of sea ice dynamics is clear, doing this explicitly would require tracking the history of a large number of leads that appear, freeze, ridge, and get split apart by consecutive sliding events along different directions at all points within the pack ice. In particular, the elastic-decohesive (Schreyer et al. 2006; Sulsky and Paterson 2011) and the elasto-brittle (Girard et al. 2011) models focus on the process of the crack formation and corresponding weakening of the sea ice without explicitly addressing their postfailure dynamics. In order for an isotropic model to be able to represent the effect of anisotropically distributed leads, the model resolution should be much smaller than the dimensions of the leads, which is generally not the case, and the rheology of sea ice should be isotropic with known scale dependence (Feltham 2008, supplemental appendix C; Taylor et al. 2006).

Here, we develop an intermediate-complexity anisotropic rheology model that allows a physically based description of discrete processes in the pack ice in such a way that it can be implemented in large-scale simulations. Our model combines several basic ideas:

(i) a description of sea ice anisotropy through the use of an orientation-dependent ice thickness distribution function (cf. Coon et al. 1998);

(ii) the assumption of coulombic shear rupture under low confinement for sea ice, based on observations (e.g., Tremblay and Mysak 1997);

(iii) the adoption of a ridging failure law for leads based on discrete element simulations (Hopkins 1998); and

(iv) a generalization of the isotropic ice redistribution model (Thorndike et al. 1975; Hibler 1980) to lead and floe ice.

Although the coulombic rheology described by a linear yield curve is simple, the effect of sea ice anisotropy can change it significantly. In particular, Hibler (2001) illustrated how the presence of several leads changes the isotropic yield curve. Furthermore, ridging that can occur simultaneously will also have an effect on the size of the yield curve envelope. For example, Tremblay and Mysak (1997) used a cutoff of the coulombic rheology at a high pressure to model ridging failure, whereas Hibler and Schulson (2000) used a more sophisticated elliptic envelope attached to the Coulomb cone to model ridging. Such approaches are, however, arbitrary and considering ridging in leads explicitly could result in a more realistic description of the yield curve.

Hibler and Schulson (2000) and Hibler (2001) considered coulombic failure in a number of leads embedded in thick ice through considering continuous deformation in both leads and thick ice. As a result, the model allowed coulombic failure in a lead with slip lines in the lead lying across the lead itself. However, satellite images usually imply a discrete failure along a crack rather than continuum failure of the whole lead, leading to the proposition that a lead can fail only along a slip line directed along the lead. Given this assumption, Wilchinsky and Feltham (2011) considered an explicit model of coulombic failure of two leads originally formed in isotropic ice.

The model we present in this paper allows a more general description of failure of anisotropic sea ice through coulombic shear rupture, ridging, and tensile failure. The yield curves will be found by combining these failure modes, with the yield curve segments described by algebraic relations between principal stress components, without the need of some of the ad hoc assumptions about a fixed yield curve shape and the corresponding flow rule inherent in some previous approaches. Although we aim at developing a model of sea ice rheology, incorporation of it into a numerical model of sea ice dynamics is beyond the scope of this present work.

The paper is structured as follows: The anisotropic sea ice failure model is presented in section 2 , which comprises discussion of our thickness distribution assumptions (section 2a); coulombic, ridging, and tensile failure modes (sections 2b-2d); ice thickness redistribution theory (section 2e); calculation of total strain rate (section $2 \mathrm{f}$ ); and a short discussion of ridging in coulombic leads (section $2 \mathrm{~g}$ ). The yield stress is presented in section 3, with separate yield curves presented for anisotropic coulombic failure (section $3 \mathrm{a})$, ridging failure (section $3 \mathrm{~b}$ ), and combined coulombic and ridging failure (section 3c). In section 4 , we discuss how to address some of the issues involved in a numerical implementation of our rheology model. Finally, in section 5, present some summary remarks and conclusions.

\section{Anisotropic sea ice failure model}

\section{a. Sea ice model}

We consider a model of sea ice consisting of areas of isotropic, polygonal ice (floes) delineated by long and 


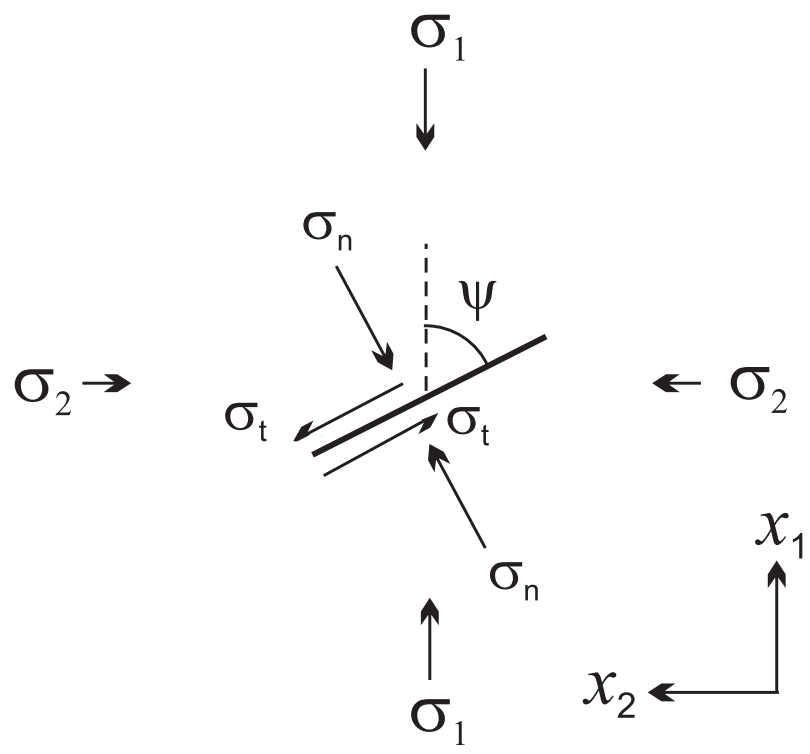

FIG. 2. Tractions on a flaw.

narrow rectangles, which are the oriented leads. Following Coon et al. (1998), in order to describe the configuration of such sea ice at a particular continuum point of the sea ice cover at a particular time, we introduce two areal ice thickness distribution functions: one for the isotropic ice in floes $g_{f}(h)$ and one for the oriented ice in leads $g_{l}(h, \psi)$, where $h$ is the ice thickness and $\psi$ is the orientation of a lead taken positive in clockwise direction from the $x_{1}$ axis (Fig. 2). For example, $g_{l}(h, \psi) d h d \psi$ is the areal fraction of sea ice in leads oriented at angles between $\psi-d \psi / 2$ and $\psi+d \psi / 2$ and having ice thickness between $h-d h / 2$ and $h+d h / 2$. By definition, the ice thickness distribution function is normalized so that $\int_{0}^{\infty}\left[g_{f}(h)+\int_{-\pi / 2}^{\pi / 2} g_{l}(h, \psi) d \psi\right] d h=1$. Note that the ice thickness distribution functions describe the sea ice state averaged over an area comprising many floes and leads and do not account for the local spatial distribution of the isotropic ice thickness and leads within this area. Although the fractional area of a lead alone does not provide any information about the lead width versus its length, we expect the lead length to be much more important in determining sea ice failure lines because a secluded short and wide lead surrounded by normally thicker isotropic ice would be less likely to fail than a narrow but long lead crossing the whole region through. In this case, for simplicity we assume that all leads have the same length and cross the whole region so that the leads area does not affect which one is going to fail.

A lead can undergo periods of deformation followed by periods of stagnation. During ridging, a lead ice thickens; during sliding, a lead tends to open due to dilatancy (Tremblay and Mysak 1997) because the lead edges are not continuously linear; and of course, during tensile opening, open water is created in a lead. During stagnation (i.e., when the lead is not actively deforming), the lead ice thickness is changed thermodynamically. These processes involve the appearance of ice of different thicknesses in a lead. If the ice of different thicknesses equally participates in ridging and sliding (parallel connection of different ice thickness regions across the lead), then lead failure is determined by the mean over all thicknesses at a particular orientation. An opposite situation is where there is a series connection of bands of different ice thicknesses across the lead and lead failure is determined by only the thinnest ice. Generally, however, ice can consist of disconnected, arbitrary regions of different thicknesses that may never extend along or across the whole lead as single bands; therefore, in considering the effect of ice thickness on lead failure, an integral thickness can be generally used weighted by a participation function for both ridging and sliding.

As the ice in a lead ridges, the newly formed ridge thickness can exceed that of the floe ice. However, because the lead is narrow, even if the ridge extends over the whole width, a failure path could easily circumvent it over the thinner floe ice. Therefore, we will assume that, whenever the ice thickness exceeds the typical floe thickness $h_{f}$, the lead ice is converted into the floe ice. The typical floe thickness can be defined to be such a thickness that ensures the same ridging force that would be obtained if the complete ice thickness distribution in the floe ice weighted by the ridging participation function was used.

Successive shear faulting and sliding at different orientations acts to make the ice cover more isotropic: for example, transecting a lead into two or more pieces and transporting them away from each other. This process does not affect the ice thickness along this orientation but makes failure along this direction harder to attain because the lead no longer extends connectedly through the whole region. A number of such events can break a lead into many disconnected pieces effectively converting the lead into the floe ice. The strength of this effect on a particular lead will be determined by the sliding rate and orientation of the affected lead relative to the sliding direction. Here, however, we will ignore this effect.

Let us define the mean thickness as $\bar{h}=\int_{0}^{\infty}\left[h g_{f}(h)+\right.$ $\left.\int_{-\pi / 2}^{\pi / 2} h g_{l}(h, \psi) d \psi\right] d h$ and the normalized thickness as $r_{h}=h / h$. Then, in the stress principal coordinate system where $x_{1}$ is the most compressive direction such that the stress principal components are $\sigma_{1}<\sigma_{2}$, coulombic, ridging, and tensile failures in the floe ice or in the leads are determined by the clockwise failure angle $\psi$ and the shear and normal tractions per unit local thickness (Fig. 2), 


$$
\left|\sigma_{t}\right|=\frac{1}{r_{h}} \tau \sin 2 \psi, \quad \sigma_{n}=\frac{1}{r_{h}}(\tau \cos 2 \psi-p),
$$

where the stress tensor invariants, pressure, and maximum shear (per unit thickness of $\bar{h}$ ) are

$$
p=-\left(\sigma_{1}+\sigma_{2}\right) / 2, \quad \tau=\left(\sigma_{2}-\sigma_{1}\right) / 2>0 .
$$

The factors $1 / r_{h}$ appear because of the continuity of the depth-integrated tractions. For a negative angle $\psi$, the plus sign at $\sin 2 \psi$ should be changed to a minus here and in the subsequent formulas. Although as the reference axis we choose the most compressive principal axis of the stress, as is seen from the SHEBA stresses plotted within a coulombic envelope (Weiss et al. 2007) the coulombic failure extends into the region of tensile stresses, so that $0<\sigma_{1}<\sigma_{2}$ is also considered. There is also a small cut off of the envelope describing tensile failure of sea ice.

\section{b. Coulombic failure}

Coulombic failure occurs along directions where the coulombic yield function $F$ expressed through local tractions attains its maximum value equal to the cohesion $\sigma_{c}$,

$$
F\left(\sigma_{n}, \sigma_{t}\right)=\left|\sigma_{t}\right|+\mu \sigma_{n}=\sigma_{c},
$$

or in terms of the stress invariants of the sea ice,

$$
F(p, \tau, \psi, r)=\frac{1}{r_{h}(\psi)}[\tau \sin 2 \psi+\mu(\tau \cos 2 \psi-p)]=\sigma_{c},
$$

where $\mu$ is the coefficient of internal friction. Clearly, the presence of cohesion is crucial for anisotropic coulombic failure as without it the effect of oriented thickness $r_{h}$ is eliminated. Tremblay and Mysak (1997) assumed zero cohesion but, comparing laboratory data with SHEBA data, Weiss et al. (2007) showed that sea ice had a nonzero cohesion that decreases by a factor between 15 and 30 when going from the laboratory scale to the geophysical scale, describing the effect of stress concentrators on fault initiation. In particular, shear at zero pressure on the geophysical scale was found to be $40 \mathrm{kPa}$, which gives $\sigma_{c}=48.8 \mathrm{kPa}$ for the internal friction coefficient $\mu=0.7$ used here. Our chosen value of $\mu$ is based on the mean value determined by laboratory observation of fault orientations at terminal failure at $-3^{\circ}$ and $-10^{\circ} \mathrm{C}$ (Schulson et al. 2006).

Generally speaking, the coulombic failure criterion cannot be satisfied simultaneously at different thicknesses of the same orientation. If, however, we assume that in our simplified lead model different ice thicknesses are distributed along the lead (parallel connection), then the width of all thickness categories across the lead is the same, and their vertical cross-sectional areas are proportional to the product of their areas and their thicknesses. If the same force were transmitted into the ice of different vertical cross-section areas, then the traction (per unit vertical cross-sectional area) in the ice of the smaller area would be higher than the traction in the ice with the larger area by a factor equal to the ratio of their areas. However, the amount of force transmitted from the floe to the lead ice is proportional to the contact area, so that there will be more force transmitted into the ice of the larger area than that of the smaller area again by a factor equal to their area ratio. In this case, these two effects cancel each other and the tractions at different ice thicknesses will be the same determined by the mean ice thickness in the lead. If all lead ice thicknesses were positioned in parallel bands along the lead (series connection), then only the thinnest ice would fail because this is where the yield function would be the highest. In a more general case, a more complicated failure pattern could be described by a coulombic failure participation function $\gamma_{s}(h)$ describing the fraction of the ice area that participates in failure. In this case, a more general normalized lead thickness should be used in the coulombic failure criterion (4),

$$
r=\frac{\int_{0}^{\infty} \gamma_{s}(h) g_{l}(h, \psi) h d h}{\bar{h} \int_{0}^{\infty} \gamma_{s}(h) g_{l}(h, \psi) d h} .
$$

The coulombic failure participation function could be taken equal to the ridging participation function discussed below.

A detailed analysis of coulombic failure of sea ice with two leads is given in Wilchinsky and Feltham (2011). Here, we consider a continuum anisotropy with regard to orientation, rather than only two ice thickness anomalies. The lead that fails through coulombic failure is found through determining where the yield function $F$ attains its maximum. At the failure line, (4) is satisfied and, because $\psi$ is calculated with regard to the most compressive stress, the factor multiplying $\tau$ is positive in (4). This means that for a fixed pressure the maximum shear $\tau$ attains its minimum at the failure line: at all other orientations a higher $\tau$ would be required for $F$ to become equal to $\sigma_{c}$. In finding the failure line, it is more convenient to determine $\tau$ from (4) and to find where it is minimum rather than finding a maximum of the yield 
function because this may exceed the cohesion for arbitrary $p$ and $\tau$.

In an isotropic case, there are two equal yield function maxima directed at the same critical angle,

$$
\psi_{c}=\frac{1}{2} \arctan (1 / \mu)
$$

on either side of the most compressive stress (Jaeger and Cook 1979; Ashby and Hallam 1986). For $\mu=0.7$ used in all our calculations, the critical flaw angle is $27.5^{\circ}$. In an anisotropic case, however, there can be a number of local yield function maxima of different magnitude. The highest maximum is where the sea ice is expected to fail first. Based on satellite images of sea ice deformation, it is natural to assume that sliding along the first lead will not be possible without another lead forming as in reality the first lead does not extend through the whole region. Generally, this second lead will form at a different angle to the highest compression axis and with a different lead traction (Wilchinsky and Feltham 2011). A skew-symmetric stress component is determined by the difference in lead tractions and their angles with regard to the axis of the highest compression. This skewsymmetric part of the stress, usually called the couple stress (e.g., Cowin 1974), would, if unopposed, result in a spin of the floes. The existence of the couple stress is caused by the simplicity of our failure model, where a homogeneous stress field is considered and the slip lines are infinite. In reality, this spin will be suppressed by the surrounding sea ice field that imposes an additional stress that counteracts the floe spin. One would expect this additional stress to be mainly concentrated at floe vertices. Similar to Wilchinsky and Feltham (2006b), we assume that the additional stress from the sea ice field can be taken into account by considering only the symmetric part of the stress tensor arising from the tractions at the slip lines. This is analogous to assuming that the additional stress arising through floe spin suppression by the surrounding sea ice field is described by a skew-symmetric stress tensor that does not contribute to work because the plastic deformation spin tensor is zero. However, although the whole sea ice stress in this case is symmetric, its constituent that determines tractions in leads is generally nonsymmetric, which must be taken into account in considering a coupled model of leads.

A nonsymmetric stress tensor can be represented through a sum of the standard symmetric part $\boldsymbol{\sigma}$, which is described by two principal values $\sigma_{1}<\sigma_{2}$, where $\sigma_{1}$ is associated with the most compressive principal stress direction, and a skew-symmetric part, whose form does not depend on the coordinate system and is described by only one parameter representing the couple-stress magnitude $\tau_{s}$,

$$
\boldsymbol{\sigma}^{\star}=\boldsymbol{\sigma}+\tau_{s}\left(\begin{array}{cc}
0 & 1 \\
-1 & 0
\end{array}\right)
$$

In this case, in its principal axes the stress field is described not only by the usual invariants of the symmetric part of the stress tensor, $p$ and $\tau$, but also by the couple stress $\tau_{s}$. Although the tensor is nonsymmetric, we consider a coordinate system that is aligned with the principal axes of the symmetric part of the stress tensor. We consider the pressure as a free parameter, whereas the shear stress and the couple stress will be determined by sea ice failure. The presence of the skew-symmetric stress does not affect normal traction on any surface, while its shear traction contribution is $\tau_{s}$. Because the first failure occurs before nonsymmetry takes effect, the first failure line is found assuming zero couple stress: namely, through finding the maximizing orientation of the yield function (4). For a fixed pressure, the first failure uniquely determines the corresponding minimum $\tau$. The second failure requires a higher shear stress, so that, in order for the tractions at the first failure line to remain at yield (3), the couple-stress magnitude has to increase to counterbalance the increase of the shear traction there. After taking into account that shear traction is taken positive in the yield function, the failure criterion (4) becomes

$$
\begin{aligned}
& F\left(p, \tau, \tau_{s}, \psi, r\right)= \\
& \left\{\begin{array}{l}
\frac{1}{r}\left[\tau \sin 2 \psi-\tau_{s}+\mu(\tau \cos 2 \psi-p)\right]=\sigma_{c}, \quad \psi>0 \\
\frac{1}{r}\left[\tau_{s}-\tau \sin 2 \psi+\mu(\tau \cos 2 \psi-p)\right]=\sigma_{c}, \quad \psi<0
\end{array}\right.
\end{aligned}
$$

Satisfaction of the failure criterion at the first failure line oriented at angle $\psi_{1}$ determines the couple stress as a function of $p$ and $\tau$,

$\tau_{s}=\left\{\begin{array}{ll}\tau\left(\sin 2 \psi_{1}+\mu \cos 2 \psi_{1}\right)-\left[\mu p+r\left(\psi_{1}\right) \sigma_{c}\right], & \psi_{1}>0 \\ \tau\left(\sin 2 \psi_{1}-\mu \cos 2 \psi_{1}\right)+\left[\mu p+r\left(\psi_{1}\right) \sigma_{c}\right], & \psi_{1}<0\end{array}\right.$.

Depending on the angle of the first failure, substituting (9) into (8) one can eliminate $\tau_{s}$ and solve for $\tau$. Minimizing $\tau$ with regard to orientation will then determine the second failure line. Similar to Tremblay and Mysak (1997), we assume no plastic spin, which implies that the two failure lines lie on opposite sides of the most compressive direction $x_{1}$.

At fixed orientations, the yield criteria (4) for the first failure line, as well as (8) and (9) for the second failure 
line, are linear in $p$ and $\tau$. Therefore, $\tau$ is a linear function of $p$ and is uniquely defined at each orientation. The sought solution for $\tau$ is found at orientations where it attains its minimum. By choosing constant $r$, one can find the standard isotropic solution for coulombic failure from (4) where failure occurs at the critical angle (6). Fixing these $\tau$ and $p$ and considering $r(\psi)$, one can use the failure criterion (4) as an equation for $r(\psi)$ so that the yield criterion is satisfied within a range of orientations. Therefore, it is clear that multiple solutions can exist, in which case we will consider only the pair of failure lines that lie closest to the critical angle either side of the largest principal stress. Because we consider a coordinate system aligned with the highest compression direction, by definition $\tau \geq 0$, and thus there are no solutions of (4) if $p<-\max _{\psi} r(\psi) \sigma_{c}$. Note that, although for $p>0$ a solution will always exist, it may require higher stresses than those required by ridging. In this case, the solution will lie outside the combined yield curve as will be discussed later.

\section{c. Ridging failure}

If the force necessary to ridge a 1-m-long ice sheet of thickness $h$ is $\Phi_{r}(h)$, then the corresponding normal traction in a lead or a slip line per unit sea ice thickness $\bar{h}$ during ridging will be $\Phi_{r}(h) / \bar{h}$, which cannot be exceeded. The form of the function $\Phi_{r}$ differs in different models. Rothrock (1975) related ice strength in convergence to the change in potential energy involved in forming a pressure ridge (and keel) so that $\Phi_{r}(h)$ is proportional to an integral over all thicknesses of the thickness squared weighted by its areal change rate. Discrete element simulations by Hopkins (1998) carried out for a particular set of material parameters reveal that a pressure ridge forms by growth of the sail until a buckling threshold is reached. The force necessary to increase sail height is determined by pushing a train of blocks over the sail surface, which, for the material parameter values adopted by Hopkins (1998), is equal to $7300 h^{3 / 2} L^{1 / 2} \mathrm{~N} \mathrm{~m}^{-1}$, where $L$ is the length of lead ice pushed into the ridge. The next phase starts when this force reaches the buckling force, $95400 h^{3 / 2} \mathrm{~N} \mathrm{~m}^{-1}$, which happens always at the same $L=L_{f}=107.7 \mathrm{~m}$. Although the ridging force varies depending on the stage of ridge formation, it is noteworthy that $\Phi_{r}$ is proportional to $h^{3 / 2}$. In all our calculations below, we will use $\Phi_{r}(h)=90 h^{3 / 2} \mathrm{kN} \mathrm{m}^{-1}, \bar{h}=3 \mathrm{~m}$, and $\sigma_{c}=48.8 \mathrm{kPa}$.

When the ridging ice in a lead consists of a distribution of thicknesses, then a ridging participation function $\gamma_{r}(h)$ [where $\left.\max \left(\gamma_{r}\right)=\gamma_{r}(0)=1\right]$ describes the fraction of ice of thickness $h$ that ridges. Again, if all ice thicknesses were distributed along the lead in a parallel connection, then they would ridge equally, and $\gamma_{r}(h)=1, \forall h$.
If all ice thicknesses were distributed in bands along the lead as in a series connection, then only the thinnest ice would ridge, and $\gamma_{r}\left(h_{\min }\right)=1$, whereas $\gamma_{r}(h)=0, \forall h>$ $h_{\min }$. To account for ridging for a more general spatial ice thickness distribution, usually a more complicated participation function is used. We will adopt a function used in isotropic models,

$$
\begin{aligned}
\gamma_{f, l}(h, \psi) & =\max \left\{\left[1-\frac{G_{f, l}(h, \psi)}{C_{1}}\right], 0\right\} \quad \text { and } \\
G_{f, l}(h, \psi) & =\frac{\int_{0}^{h} g_{f, l}\left(h^{\prime}, \psi\right) d h^{\prime}}{\int_{0}^{\infty} g_{f, l}\left(h^{\prime}, \psi\right) d h^{\prime}}, \quad G<C_{1}
\end{aligned}
$$

(Thorndike et al. 1975), where only the thinnest fraction $C_{1}$ of the lead ice is ridged (typically $C_{1}$ is set to 0.15 ). For brevity, we use subscripts $f$ and $l$ to point at functions for the floe ice and the lead ice, respectively, keeping in mind that the floe ice characteristics are orientation independent. The proportion of the ice ridged within the fraction $C_{1}$ gradually decreases from 1 to 0 as the thickness approaches the thickest ice available for ridging. This participation function was introduced for isotropic sea ice as a whole. We will, however, adopt it here for modeling ridging within a single lead. In this case, the normal traction (per unit mean ice thickness $\bar{h}$ ) necessary to ridge the lead ice is the pressure ridging force integrated over all participating thicknesses and divided by the mean thickness,

$$
F_{r}(\psi)=\frac{\int_{0}^{\infty} \Phi_{r}(h) \gamma_{l}(h, \psi) g_{l}(h, \psi) d h}{\bar{h} \int_{0}^{\infty} \gamma_{l}(h, \psi) g_{l}(h, \psi) d h} .
$$

Therefore, ridging in a lead implies

$$
\tau \cos 2 \psi-p=-F_{r}(\psi)
$$

where the right-hand side is the normal traction in the sea ice given by (1) with $r_{h}=1$, and the minus at $F_{r}$ appears because compressive stress is negative. Because all lead ice thicker than the floe ice thickness $h_{f}$ is redistributed into floe ice, if ridging occurs at a particular orientation, it would occur in a lead, unless there is no lead at this orientation. The orientation at which ridging occurs is determined by minimizing $(\tau \cos 2 \psi-p) / F_{r}(\psi)$ with regard to orientation. Because the factor at the pressure is negative and this expression is higher than -1 along nonfailure orientation, for a fixed $\tau, p$ attains its minimum at failure lines if (13) is assumed to hold at all orientations. 
Because $F_{r}$ depends on orientation, this minimum depends on both $p$ and $\tau$. Ridging failure requires that at this minimizing orientation (13) holds, relating $p$ to $\tau$. Ridging can also occur in the floe ice, in which case $F_{r}$ should be suitably redefined in (12) replacing all functions relative to the lead to those relative to the floe. Similarly to the coulombic failure, (13) is linear in both $p$ and $\tau$, so that, at a fixed orientation, $p$ is uniquely defined for a given $\tau$. At the same time, $F_{r}$ can be chosen in such a way that (13) is satisfied simultaneously at more than two orientations, in which case we choose only the two closest to the stress principal axes. Because $\tau<0$ cannot be considered, this puts limits on values of $p$ for which solutions exist.

\section{d. Tensile failure}

Experiments have shown the existence of sea ice tensile strength (Richter-Menge and Jones 1993; Dempsey et al. 1999) as well as a high sensitivity of the ice tensile strength to its temperature and structure (e.g., crack density). During the Sea Ice Mechanics Initiative study on a 1.42-mthick floe, Lewis and Richter-Menge (1998) recorded tensile stresses up to $80 \mathrm{kPa}$. The presence of the tensile strength is reflected as a cutoff of the coulombic yield curve in the region of negative pressures (Weiss et al. 2007). If we assume that sea ice tensile strength is given by $\Phi_{o}(h) \propto h$, then, similar to ridging, we can write the normal traction necessary for tensile failure,

$$
F_{o}(\psi)=\frac{\int_{0}^{\infty} \Phi_{o}(h) \gamma_{l}(h, \psi) g_{l}(h, \psi) d h}{\bar{h} \int_{0}^{\infty} \gamma_{l}(h, \psi) g_{l}(h, \psi) d h} .
$$

Therefore, opening of a lead due to tensile failure implies

$$
\tau \cos 2 \psi-p=F_{o}(\psi) .
$$

The opening lead or direction of new lead formation in the floe ice is then found by maximizing $(\tau \cos 2 \psi-p) /$ $F_{o}(\psi)$ with regard to orientation. Similar to ridging, we consider only two opening leads at most and, if tensile failure occurs in the floe ice, $F_{o}$ should be suitably redefined with the corresponding functions relative to the floe ice. Note that the total number of both opening and ridging leads is limited to two because there cannot be simultaneous opening and closing of two pairs of leads.

\section{e. Ice thickness redistribution}

Ridging makes the ice thicker, whereas sliding along a failure line creates opening due to dilatancy. Similarly, tensile failure leads to opening and open water production. All these processes change the ice thickness distribution. The rate of change of ice area of thickness $h$ per unit convergent deformation in the floe and the lead is (Thorndike et al. 1975; Hibler 1980)

$$
\omega_{f, l}(h, \psi)=\frac{-a_{f, l}(h, \psi)+\int_{0}^{\infty} \beta\left(h^{\prime}, h\right) a_{f, l}\left(h^{\prime}, \psi\right) d h^{\prime}}{1-\int_{0}^{\infty} \int_{0}^{\infty} \beta\left(h^{\prime}, h^{\prime \prime}\right) a_{f, l}\left(h^{\prime}, \psi\right) d h^{\prime} d h^{\prime \prime}},
$$

where

$$
a_{f, l}(h, \psi)=\frac{\gamma_{f, l}(h, \psi) g_{f, l}(h, \psi)}{\int_{0}^{\infty} \gamma_{f, l}\left(h^{\prime}, \psi\right) g_{f, l}\left(h^{\prime}, \psi\right) d h^{\prime}}
$$

is the normalized distribution of ridging ice and the transfer function $\beta\left(h_{1}, h_{2}\right)$ defines the area of ice of thickness $h_{2}$ produced by ridging of a unit area of ice of uniform thickness $h_{1}$. A standard choice of the transfer function $\beta\left(h^{\prime}, h\right)$ introduced by Hibler (1980) corresponds to the buildup of a ridge of triangular shape: uniform ice is redistributed between twice the thickness of the ice being ridged and a maximum ridge thickness of $2 \sqrt{H^{*} h}$,

$$
\beta\left(h, h^{\prime}\right)=\frac{1}{2\left(H^{*}-h\right)}, \quad 2 h \leq h^{\prime} \leq 2 \sqrt{H^{*} h} .
$$

Note that the ridge width and not its thickness depends on the area of the ridged ice. Therefore, if a ridge exceeds the mean floe thickness, then it occurs regardless of the amount of deformation, so that in this case the lead ice that is transferred to the floe ice can consist of a range of thicknesses with the lower boundary being the characteristic floe thickness $h_{f}$. Because we assume that ice in the lead cannot be thicker than the mean floe ice, we have $g_{l}(h, \psi)=a_{l}(h)=0, \forall h>h_{f}$. By definition,

$$
\int_{0}^{\infty} a_{f, l} d h=1 \Rightarrow \int_{0}^{\infty} \omega_{f, l} d h=-1 .
$$

The evolution of the ice thickness distribution is given by

$$
\frac{d g_{f, l}}{d t}=\Omega_{f, l}+f_{h}-g_{f, l}\left[\operatorname{div}(\mathbf{v})+\frac{\partial f_{v}}{\partial h}\right]
$$

(Thorndike et al. 1975), where $d / d t$ is the total time derivative (i.e., it includes rotation as well as advection); $\mathbf{v}$ is the velocity vector; $\Omega_{f, l}$ are ice thickness redistribution functions in the floe and leads due to ridging, sliding, and 
opening; $f_{v}$ is the vertical freeze-melt rate, and $f_{h}$ is the lateral freeze-melt rate (added by Hibler 1980). The term including the divergence describes the effect of ice fraction decrease if the region containing it expands. The sea ice redistribution functions $\Omega_{f, l}$ account for the amount of ridging and opening as a function of deformation in leads,

$$
\begin{aligned}
\Omega_{l}= & \chi_{r}(\psi) \dot{\xi}_{r}(\psi) \omega_{l}(h, \psi)+\chi_{s}(h, \psi) \dot{\xi}_{s}(\psi) \delta \\
& +\chi_{o}(h, \psi) \dot{\xi}_{o}(\psi), \quad h \leq h_{f} \quad \text { and } \\
\Omega_{f}= & \chi_{r}(\psi) \dot{\xi}_{r}(\psi) \omega_{f}(h) \\
& +\int_{-\pi / 2}^{\pi / 2} \chi_{r}(\psi) \dot{\xi}_{r}(\psi) \omega_{l}(h, \psi) d \psi, \quad h>h_{f},
\end{aligned}
$$

where $\dot{\xi}_{r}(\psi)$ is the rate of extension perpendicular to a ridging lead (negative in ridging and zero otherwise), $\dot{\xi}_{s}(\psi)$ is the rate of shearing along the lead if coulombic failure occurs, and $\delta$ is the rate of dilatancy per unit shearing, so that $\dot{\xi}_{s} \delta$ is the rate of opening of the shearing lead. The opening rates $\dot{\xi}_{o}(\psi)$ under tensile failure (positive in opening and zero otherwise) are also included. The magnitudes of the deformation rate functions $\dot{\xi}_{r, s, o}$ do not affect the stresses because the yield is plastic and are determined from momentum equations. The functions $\chi_{r}(\psi)$ and $\chi_{s, o}(h, \psi)$ are the ridging, sliding, and tensile failure Dirac delta function, so defined that $\chi_{r}(\psi)=0, \forall \psi \neq \psi^{r}$, and $\int_{\psi^{r}-d \psi}^{\psi^{r}+d \psi} \chi_{r}\left(\psi^{\prime}\right) d \psi^{\prime}=1$, as well as similarly $\chi_{s, o}(h, \psi)=0, \forall \psi \neq \psi^{s, o}$ or $\forall h>0$, and $\int_{0}^{d h} \int_{\psi^{s, o}-d \psi}^{\psi^{s, o}+d \psi} \chi_{s, o}\left(h^{\prime}, \psi^{\prime}\right) d \psi^{\prime} d h^{\prime}=1$, where $\psi^{r}, \psi^{s}$, and $\psi^{o}$ are any angles where ridging, coulombic, and tensile failure occur, respectively. The Dirac delta functions are used to incorporate finite opening/closing rates at a single orientation into the continuous ice thickness distribution function. The first term in function $\Omega_{f}$ describes the effect of possible ridging of the floe ice, whereas the second term describes the effect of transfer into the floe ice of the lead ice that during ridging becomes thicker than the floe ice. Due to normalization (19),

$$
\begin{aligned}
\operatorname{div}(\mathbf{v}) & =\int_{0}^{\infty}\left(\Omega_{f}+\int_{-\pi / 2}^{\pi / 2} \Omega_{l} d \psi\right) d h \\
& =2 \dot{\xi}_{s} \delta+\dot{\xi}_{r, o}^{(1)}\left(\psi_{1}^{r, o}\right)+\dot{\xi}_{r, o}^{(2)}\left(\psi_{2}^{r, o}\right),
\end{aligned}
$$

Where, if failure occurs along fewer directions, the corresponding terms should be ignored.

Note that if, at a particular orientation there is no lead (i.e., its area is zero), ridging of the floe ice perpendicular to this direction redistributes only the floe ice. However, if coulombic failure and sliding occur along this direction (or tensile failure and opening), the dilatancy (or opening) affects the lead ice thickness by increasing its area from zero proportional to the shearing rate. As a continuum ice thickness distribution function is used, closing-opening of a single-orientation lead results in an infinite rate of ice thickness redistribution. In this case, a ridging lead would immediately thicken and either another lead will start failing or, if no other lead ready to fail is present, the original lead will turn into the floe ice. Similarly, sliding of a lead accompanied by opening will immediately result in the mean lead thickness becoming zero if thermodynamic growth is disregarded, which will make it more susceptible to ridging, which is discussed later. In numerical treatments that use discrete ice categories with regard to thickness and orientation, deformation processes in one orientational category will have a finite rate.

\section{f. Strain rate}

To write down the strain rate tensor, we assume that coulombic failure and the corresponding sliding occurs always along two failure lines. Similar to Tremblay and Mysak (1997), we assume no spin due to sliding, which is possible only if the failure lines positioned are either side of the most compressive axis and their sliding rates are equal. For certain ice thickness distributions, the yield criterion (4) can be satisfied at more than two directions. In this case, for simplicity we choose only those two directions that lie closest to the isotropic critical failure angles (6). If we denote the angles of our two coulombic failure lines as $\psi_{1}^{s}$ and $\psi_{2}^{s}$, then in coordinate systems aligned with the failure lines the strain rate tensor contributions at unit shear rate along them are

$$
\dot{\mathbf{e}}_{1,2}^{s}=-\operatorname{sgn}\left(\psi_{1,2}^{s}\right)\left(\begin{array}{ll}
0 & 1 \\
1 & \delta
\end{array}\right),
$$

where $\delta$ is the dilatancy rate. Rotating these contributions into the principal stress coordinate system and adding them together gives the total sliding contribution to the strain-rate tensor,

$$
\dot{\mathbf{e}}^{s}\left(\psi_{1}^{s}, \psi_{2}^{s}\right)=\left[\begin{array}{cc}
-2 \cos \left(\psi_{1}^{s}+\psi_{2}^{s}\right) \sin \Delta \psi^{s}+\delta\left(\sin ^{2} \psi_{1}^{s}+\sin ^{2} \psi_{2}^{s}\right) & \left(2 \sin \Delta \psi^{s}+\delta \cos \Delta \psi^{s}\right) \sin \left(\psi_{1}^{s}+\psi_{2}^{s}\right) \\
\left(2 \sin \Delta \psi^{s}+\delta \cos \Delta \psi^{s}\right) \sin \left(\psi_{1}^{s}+\psi_{2}^{s}\right) & 2 \cos \left(\psi_{1}^{s}+\psi_{2}^{s}\right) \sin \Delta \psi^{s}+\delta\left(\cos ^{2} \psi_{1}^{s}+\cos ^{2} \psi_{2}^{s}\right)
\end{array}\right]
$$


where $\Delta \psi^{s}=\psi_{2}^{s}-\psi_{1}^{s}>0$ is the difference between the failure angles. If we define the strain rate tensor invariants as

$$
\dot{e}_{I}=\frac{1}{2} \dot{e}_{i i}, \quad \dot{e}_{I I}=\left(\frac{1}{2} \dot{e}_{i j}^{*} \dot{e}_{i j}^{*}\right)^{1 / 2},
$$

where the asterisk denotes a traceless part, then

$$
\dot{e}_{I}^{s}=\delta, \quad \dot{e}_{I I}^{s}=\left|\delta \cos \Delta \psi^{s}+2 \sin \Delta \psi^{s}\right| .
$$

As is expected half the divergence $\dot{e}_{I}^{s}$ due to sliding along coulombic lines is equal to the dilatancy rate $\delta$. The maximum shear deformation rate $\dot{e}_{I I}^{s}$ has two components. The first one is related to the nonuniformity of dilatancy with regard to orientation and it disappears if dilatancy is equal in perpendicular directions $\left(\Delta \psi^{s}=\pi / 2\right)$ and is maximum when it is unidirectional $\left(\Delta \psi^{s} \rightarrow 0\right)$, leading to a maximum difference in deformation in the principal directions. The second contribution comes from sliding along the two failure lines. Because the shear along the two failure lines is the same in magnitude but opposite in sign, the closer the failure lines are aligned with each other, the more shear strain rate from one line becomes compensated by the other, canceling each other when the failure lines become parallel and, vice versa, doubling when they become perpendicular.

Strain rate contributions due to ridging and opening are quite similar, but with opposite signs; therefore, we will discuss only ridging. In considering ridging failure, we assume that it can occur along one or two failure lines. If there are more possible failure lines where the ridging yield criterion (13) is satisfied, we disregard them for simplicity choosing the closest to the stress principal axes. If the failure occurs along $\psi^{r}$, which could be in either floe ice or in the lead, then it is described by a uniaxial contractional deformation in the perpendicular direction, which after rotation into the principal stress coordinate system gives the corresponding strain rate contribution at unit ridging rate as

$$
\dot{\mathbf{e}}^{r}\left(\psi^{r}\right)=\left(\begin{array}{cc}
\sin ^{2} \psi^{r} & \frac{1}{2} \sin 2 \psi^{r} \\
\frac{1}{2} \sin 2 \psi^{r} & \cos ^{2} \psi^{r}
\end{array}\right) .
$$

If ridging occurs along two lines, then a similar contribution comes from the second ridging line, but with a different ridging rate $\dot{\xi}_{r}$.

Adding the contributions from all failure regimes described by different rates, we obtain the cumulative strain rate in the form

$$
\dot{\mathbf{e}}=\dot{\xi}_{s} \dot{\mathbf{e}}^{S}\left(\psi_{1}^{S}, \psi_{2}^{S}\right)+\dot{\xi}_{r, o}^{(1)} \dot{\mathbf{e}}^{r, o}\left(\psi_{1}^{r, o}\right)+\dot{\xi}_{r, o}^{(2)} \dot{\mathbf{e}}^{r, o}\left(\psi_{2}^{r, o}\right) .
$$

A contribution from opening due to tensile failure is similar to the ridging contribution, but with a positive rate. In cases when failure occurs along fewer directions, the corresponding terms should be ignored.

\section{g. Ridging failure of a coulombic lead}

As is seen in Fig. 1, frequently a coulombic failure is followed by a ridging failure of the same lead. Clearly, as a lead is subject to dilatancy, shearing along a failing lead results in open water production and reduction of the mean thickness of the lead. The open water will quickly freeze over, and thermodynamic processes in winter increase the lead thickness. The lead thickness therefore evolves by the balance between dilatancy, thermodynamic, and ridging thickening. The participation function (10) implies that ridging occurs only in the thinnest $15 \%$ of the lead area. The opening rate for a lead is $\dot{\xi}_{s} \delta$, where we take $\delta=\tan 10^{\circ}=0.18$ (Tremblay and Mysak 1997). Stern and Lindsay (2009) analyzed Radarsat data and found that generally the deformation rate can vary within two orders of magnitude: from 0.001 to $0.1 \mathrm{day}^{-1}$. The mean deformation rate increases with decreasing scale, and attains its maximum of $0.02 \mathrm{day}^{-1}$ in winter and $0.07 \mathrm{day}^{-1}$ in summer at a $1-\mathrm{km}$ scale. Therefore if we take the winter shear rate scale as $\dot{\xi}_{s}=0.01 \mathrm{day}^{-1}$, the opening rate will be $1.8 \times 10^{-3}$ day $^{-1}$, which is $18 \%$ day $^{-1}$ of a lead with a fractional area of 0.01. In this case, after a deformation event it is likely that the ridging stress of the lead will be determined by the opened area of water only, whose initial freezing could at best produce only light nilas up to $10-\mathrm{cm}$-thick in one day. In this case the ridging force required to ridge the lead will be minimal in comparison to other leads, and the new lead will preferentially fail through ridging.

\section{Yield stress}

Given a prescribed pressure $p$, depending on the maximum shear stress the sea ice can fail coulombically, through ridging-tensile opening, or two failure modes can occur simultaneously. For a range of shear stresses, no failure will occur at all, describing a subyield regime. At a given pressure, the maximum shear stress cannot lie outside of the range determined by the different failure regimes. Similarly, where failure is possible the pressure cannot lie outside of its own range. Generally speaking, when occurring simultaneously, coulombic and ridging/ tensile failure can affect different leads. In this case, the yield curve will be a combination of yield curves determined by coulombic and ridging failures. The tensile cut off of the coulombic yield curve is relatively small (Weiss et al.2007); therefore, below it will be ignored for simplicity. 


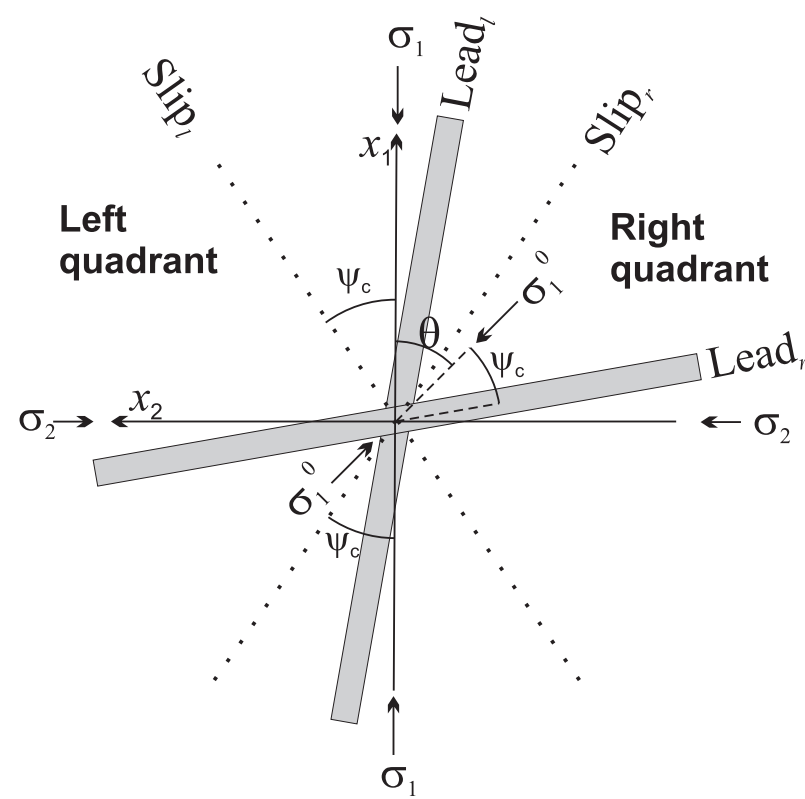

FIG. 3. Rotation of two leads originally formed at the critical slip line angles with regard to the direction of the highest compression by an angle $\theta$. Vectors $\boldsymbol{\sigma}_{1}^{0}$ show the initial direction of the highest compression leading to formation of these leads.

\section{a. Yield stress in coulombic failure}

When sea ice is isotropic, coulombic failure occurs at critical angles $\psi_{c}$ given by (6) that do not depend on pressure, and therefore the maximum shear stress $\tau$, determined by (4) with $r=1$, is a linear function of pressure $p$. However, when sea ice is anisotropic, the two failure angles determined by (4) and (9) are dependent on the anisotropy as well as $p$ and the corresponding yield curve can have a more complicated shape. To illustrate this, similar to Wilchinsky and Feltham (2011) let us for a moment neglect the ridging failure and consider how the yield curve changes when two leads originally positioned at $\pm \psi_{c}$ are rotated clockwise with regard to the most compressive direction by an angle $\theta$ (see Fig. 3). We set the normalized lead thickness to be $r=0.1$. Figure 4 shows the yield curves $\tau(p)$ for different $\theta$ and $\tau(\theta)$ for different $p$. It can be seen that, at some angles of rotation, as $p$ changes, the angle of the yield curve $\tau(p)$ also changes. The reason for this is clearer from inspecting the dependence of $\tau$ on $\theta$, which consists of several branches. The leftmost branch, which for small $p$ is bounded by $\theta=\psi_{c}$, corresponds to the two leads failing simultaneously. The angle $\theta=\psi_{c}$ corresponds the left lead leaving the left quadrant and entering the right quadrant (Fig. 3), so that for small $p$ this branch is followed by another where the floe ice fails in the left quadrant (negative $\psi$ ), whereas the leftmost lead fails in the right quadrant (positive $\psi$ ). This branch has a local minimum because the shear stress decreases as the lead angle approaches the critical angle. For higher pressures, these two branches are separated by a flat line describing isotropic failure of the floe ice because the two leads are too far away from the critical angle where failure is the most likely. This flat segment widens as the pressure increases because, as can be seen from the yield criterion (4), an increase in $p$ leads to a larger decrease of the yield function for smaller ice thicknesses $r$ and to a smaller decrease for larger ice thicknesses. Therefore, as the pressure increases, in the vicinity of $\theta=\psi_{c}$ the yield function at the critical angle of the floe ice can exceed the yield function at the lead causing switching of failure from the lead to the thicker, floe ice. This change determines the corresponding change of the yield curve angle for $\tau(p)$. Similarly, $\theta=\pi / 2-\psi_{c}$ is the angle where the rightmost lead leaves the right quadrant and enters the left quadrant, and it can fail there for low pressures leading to lowering of the shear stress. This branch then descends as the lead approaches the critical angle. For higher pressures, however, failure in the left quadrant remains in the floe ice and, as the remaining (originally left) lead in the right quadrant rotates farther away from the critical angle, failure switches to the floe ice again with the corresponding flat branch widening for the same reasons as described earlier.

This behavior is also reflected in the direction of the more convergent principal strain rate axis $\psi_{e}^{s}$. This is positioned in the center of the two active leads. Initially, at small rotation angles $\theta$, because the failure occurs in the leads the strain rate direction follows the leads. Then, however, when the left lead approaches the axis of the highest compression while the right lead rotates farther away from the critical angle, for higher pressures the thick, floe ice will preferentially fail so that the strain rate direction switches back to $x_{1}$. After further rotation, the left lead in the right quadrant approaches the critical angle and becomes active again, whereas in the left quadrant the floe ice fails at the critical angle, so that in this case the strain rate direction, which is in the middle, switches to the left quadrant and becomes negative. As rotation progresses, it follows the rotation angle but at a half rate. At $\theta=\pi / 2-\psi_{c}$ the right lead enters the left quadrant and at small pressures the two leads become active, and because the lead in the left quadrant is at $-\pi / 2$ while that in the right quadrant is at $\pi / 2-2 \psi_{c}$ the strain rate direction again switches to the left quadrant. For higher pressures however, the failure lines remain the same until in the right quadrant they switch from the lead to the floe ice. The second strain rate invariant is independent of the lead orientation; rather, it depends on the angle between the leads with two different contributions as was discussed earlier: from the dilatancy 

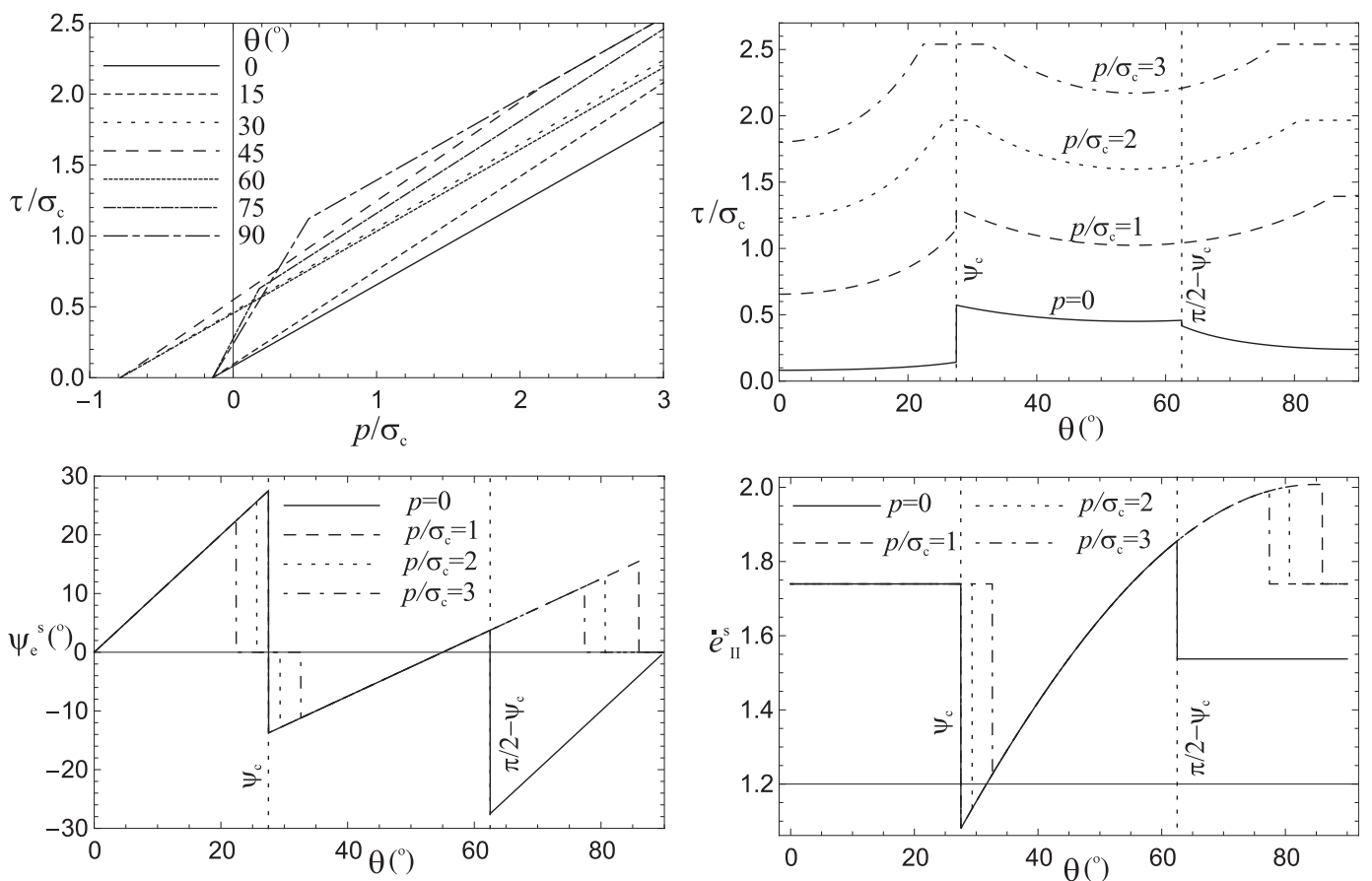

FIG. 4. Yield curves in the principal stress coordinates for coulombic failure with $r=0.1$ and $\delta=\tan 10^{\circ}$. (top left) Maximum shear stress $\tau$ as a function of pressure $p$ for different angles of rotation $\theta$ of two leads with regard to the largest compression direction. (top right) Maximum shear stress $\tau$ as a function of the rotation angle $\theta$ for different pressures $p$. (bottom left) The angle of the more convergent principal direction of the strain rate tensor vs $\theta$. (bottom right) Second strain rate invariant $\dot{e}_{I I}^{s}$ vs $\theta$.

and shear along them. The taken dilatancy angle of $10^{\circ}$ determines $\delta=\tan 10^{\circ}=0.18$, so that the contribution from sliding along the lines largely determines the maximum shear rate. Therefore, the farther away the two leads are from each other, the higher is the maximum shear strain rate, which is reflected in Fig. 4 (bottom right).

\section{b. Yield stress in ridging failure}

The yield criterion for ridging failure is given by (13), which determines a $\tau(p)$ relationship. However, because it is singular at $\psi=\pi / 4$, it is more convenient to consider an inverted relationship $p(\tau)$,

$$
p=\tau \cos 2 \psi+F_{r}(\psi)
$$

Therefore, here, instead of minimizing the maximum shear, we minimize the pressure with regard to orientation to determine which direction is going to fail by ridging. If the sea ice is isotropic $\left[F_{r}(\psi)=\right.$ const $]$ and $\tau \neq$ 0 , then the ridging failure angle does not depend on $\tau$ and is perpendicular to the most compressive direction, $\psi=\pi / 2$. If $\tau=0$, then ice can fail equally at any direction. For anisotropic sea ice, however, the minimum of the right-hand side is $\tau$ dependent, so that the ridging failure angle depends on the maximum shear stress. If the minimizing angle $\psi^{r}(\tau)$ is found, then, if it does not change for a range of $\tau$, within this range the yield curve is a straight line intersecting the $p$ axis at $p=F_{r}\left(\psi^{r}\right)$ and making an angle with this axis of $\arctan \left(1 / \cos 2 \psi^{r}\right)$. This (anticlockwise) angle of the yield line from the $p$ axis gradually varies from $\pi / 4$ at $\psi^{r}=0$ through $\pi / 2$ at $\psi^{r}=$ $\pi / 4$ up to $3 \pi / 4$ at $\psi^{r}=\pi / 2$.

To illustrate this, let us consider an idealized scenario with only two leads present, which are aligned with the principal axes (Fig. 5). We consider leads of uniform thickness, so that the thicker lead (higher $r$ ) is harder to ridge than the thinner lead (lower $r$ ). The same can be achieved if for this ridging failure we redefine $r=F_{r}$ as a measure of the strength of the lead opposition to ridging. For compression under confinement, the leads do not fall into the lead failure range and therefore will not fail coulombically (Wilchinsky and Feltham 2011), so that the coulombic yield curve is determined by standard isotropic failure in the floe ice. Let us consider this coulombic failure at the state where the most compressive stress $\sigma_{1}$ is such that lead 2 (lying perpendicular) fails. This stress cannot have a higher magnitude because of this ridging failure. Because in this state the sea ice also fails coulombically, this stress state is 


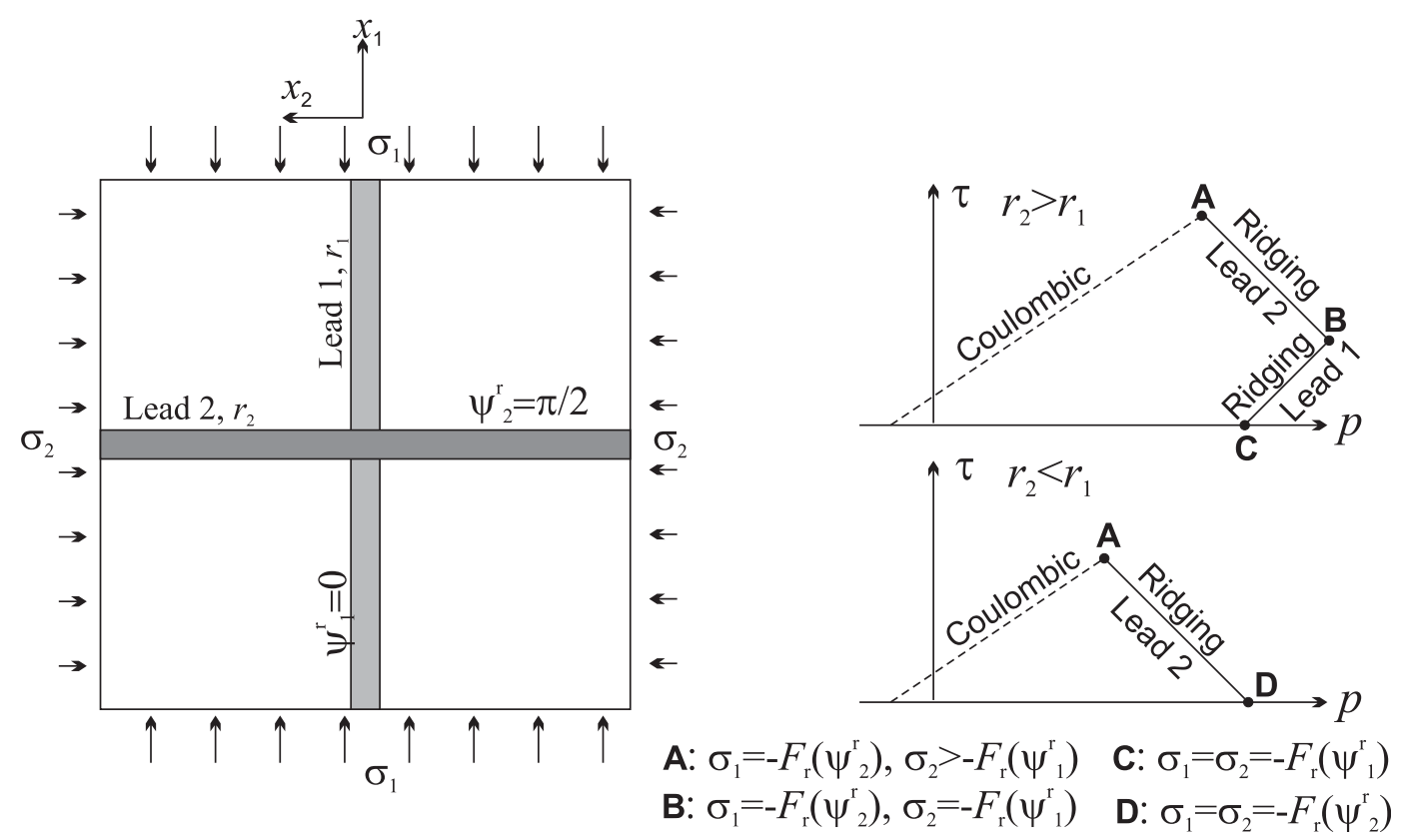

FIG. 5. (left) Two leads aligned with the stress principal axes. (right) The schematic representation of the yield curves corresponding to the two cases of relative lead thickness.

described by point A on the plots in Fig. 5. If the least compressive stress $\sigma_{2}$ decreases (becomes more compressive), the maximum shear stress is reduced while the pressure is increased by the same amount, describing a southeast $\left(-45^{\circ}\right)$ trajectory in $(p, \tau)$ coordinates. Along this branch, the sea ice cannot fail coulombically anymore, but, since $\sigma_{1}$ remains the same, lead 2 is still in ridging failure, so that the stress remains on the yield curve now determined only by ridging failure of lead 2.

If lead 2 is thicker than lead $1\left(r_{2}>r_{1}\right)$, then decreasing $\sigma_{2}$ further eventually leads to this stress reaching the ridging stress of lead 1 described by point $\mathrm{B}$ on the top plot. At this state $\sigma_{1}<\sigma_{2}$ and the thinner is lead 1 relative to lead 2 , the shorter will be the $[A, B]$ branch. No principal stress can be decreased any further because they are bounded by the ridging stresses. However, if now the most compressive stress $\sigma_{1}$ is increased (becomes less compressive), then the pressure and the maximum shear stress will decrease by the same amount, describing a southwest $\left(-135^{\circ}\right)$ trajectory in the $(p, \tau)$ coordinates, whereas lead 1 will remain in ridging failure. Therefore, the stress remains on the yield curve, now determined by ridging of lead 1 , whereas point $B$ describes switching from ridging of lead 2 to lead 1 . Here, $\sigma_{1}$ can increase even further until it reaches $\sigma_{2}$ describing uniform compression $\left(\sigma_{1}=\sigma_{2}\right.$; point C). If the leads are of equal thickness, $r_{1}=r_{2}$, then point $\mathrm{B}$ coincides with point $\mathrm{C}$.
If lead 1 is thicker than lead $2\left(r_{1}>r_{2}\right)$, then, starting again from point $\mathrm{A}$ describing simultaneous coulombic and ridging failure of lead $2, \sigma_{2}$ can be decreased until uniform compression ( $\sigma_{1}=\sigma_{2}$; point D). Simultaneous ridging failure of both leads cannot, however, be achieved because this would require $\sigma_{1}>\sigma_{2}$, which would violate the choice of our coordinate system (because it would make $x_{2}$ the most compressive direction).

Changing the angles of the leads would change the angles of the yield lines, whereas changing the lead thicknesses will change the position of intersection points. Because we choose $x_{1}$ to be the most compressive direction, we always have $\tau \geq 0$, so that the yield curve is plotted only in the upper semiplane. If one wanted to extend the yield curve into the lower semiplane, then for $r_{2}>r_{1}$ the top plot in Fig. 5 should be appended by the bottom plot $\left(r_{2}<r_{1}\right)$ reflected with regard to the $p$ axis because, as $\tau$ turns negative, $x_{2}$ becomes the most compressive direction and the leads exchange positions relative to the most compressive direction. Similarly, if $r_{1}<r_{2}$ one would append the top plot, reflected about the $p$ axis, to the bottom plot.

\section{c. Combined yield curves}

In analyzing coulombic and ridging failure separately, we have considered only two leads. Considering an arbitrary lead thickness distribution makes interpretation of the result difficult because of a high degree of freedom. Therefore, here we consider an intermediate-complexity 


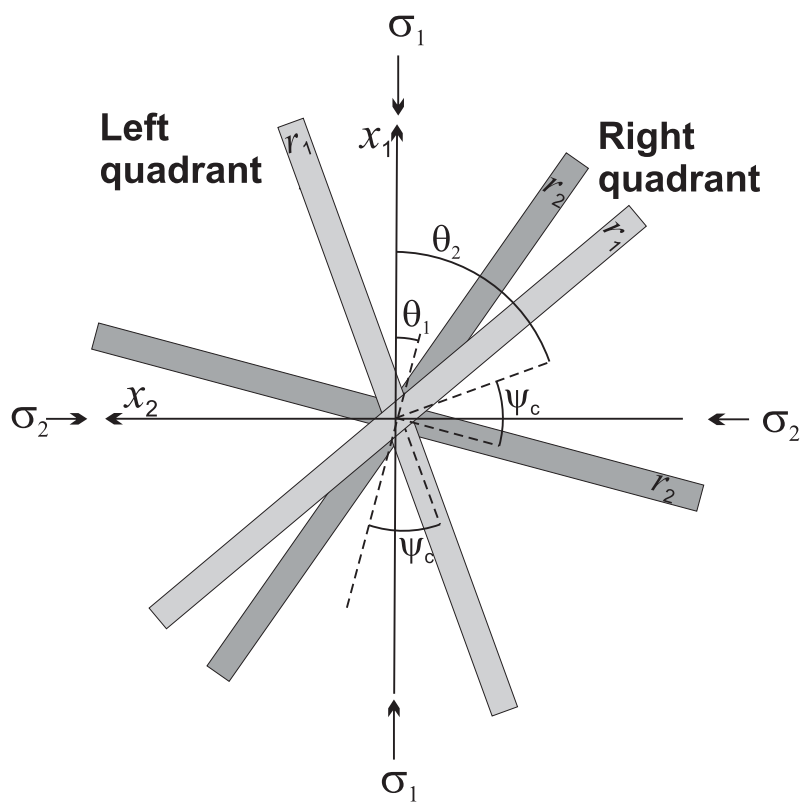

FIG. 6. Two pairs of leads formed in floe ice under different conditions at different times so that their angles with respect to the most compressive principal stress and thicknesses differ.

scenario of two pairs of leads separated by an angle of $2 \psi_{c}$ and having different, uniform thicknesses $r_{1}$ and $r_{2}$ (Fig. 6). This models the result of two incidents of lead formation in the floe ice where the time difference had led to a difference in their thickness because of freezing. Even when a pair of leads is present, under special conditions a new pair can still form in the floe ice as is seen from Fig. 4. The pairs are rotated clockwise by $\theta_{1}<\theta_{2}$ with regard to the most compressive axis, respectively. This situation allows four degrees of freedom $\left(\theta_{1}, \theta_{2}, r_{1}\right.$, and $\left.r_{2}\right)$, and we restrict ourselves by fixing the second pair around the least compressive direction $\left(\theta_{2}=\pi / 2\right)$ while considering its thickness to be half of the floe ice thickness $\left(r_{2}=0.5\right)$ where the floe ice thickness is $\bar{h}=3 \mathrm{~m}$. Figure 7 shows the combined yield curves when the first pair is positioned around the most compressive direction $\left(\theta_{1}=0\right)$ and its thickness varies and when its thickness is fixed to $r_{1}=0.3$ and its angle $\theta_{1}$ varies. It can be seen that, as the thickness of the first pair increases while its position is fixed at $\theta_{1}=0$, the maximum shear stress at coulombic failure naturally grows. This is because these leads are positioned at the critical angles $\pm \psi_{c}$ so that they will fail coulombically and making them thicker increases the required stress. Considering the ridging branch of the yield curve, then at small $r_{1}$ the first pair is easy to ridge and since its angle is less than $\pi / 4$ the yield curve angle is negative. As $r_{1}$ increases, the first pair becomes harder to ridge and, because the second pair is positioned closer to the least compressive direction (the preferential direction for ridging failure), ridging failure at the second pair becomes predominant.

Similarly, because $r_{1}=0.3$ is fixed, as the first pair of leads rotates closer to the least compressive direction, the first pair becomes easier to ridge compared to the second pair so that the yield line becomes directed more anticlockwise relative to the $p$ axis. Moreover, because the yield curve angle is determined by the ridging lead angle the ridging branch rotates anticlockwise as the right lead approaches $x_{2}\left(\theta_{1} \rightarrow \pi / 2-\psi_{c}=62.5^{\circ}\right)$. It can be seen that at $\theta_{1}=75^{\circ}$ the branch rotates back as the ridging lead deviates from the direction of preferential ridging $x_{2}$. Furthermore, as the first pair rotates away from the critical angles $\pm \psi_{c}$, which are the preferential directions of coulombic failure, the floe ice starts failing, leading to formation of steep-angle segments on the yield curve. It is interesting to note that the plotted yield curves are characterized by tensile and compressive stresses of a similar magnitude, which concurs with a coulombic envelope around the SHEBA data (Weiss et al. 2007).
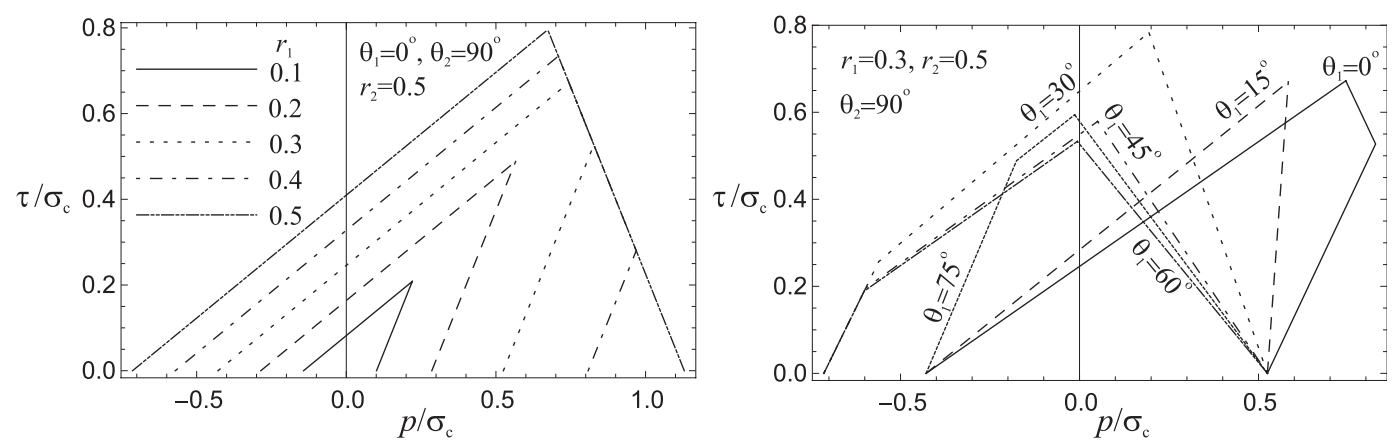

FIG. 7. Combined yield curves for coulombic and ridging failure. (left) For fixed positions of the two lead pairs at $\theta_{1}=0, \theta_{2}=\pi / 2$ and variation of the thickness of the first pair $r_{1}$ while $r_{2}=0.5$. (right) For fixed thickness $r_{1}=0.3$, $r_{2}=0.5$ and variation of the position of the first pair $\theta_{1}$ while $\theta_{2}=\pi / 2$. Mean floe thickness is $\bar{h}=3 \mathrm{~m}$. 


\section{Discussion}

The main advantages of the anisotropic sea ice rheology presented here over standard, isotropic models are (i) our model takes account of observations implying that failure in the pack ice occurs along anisotropically distributed discrete lines and (ii) our rheology is explicitly calculated from failure at these lines, at which coulombic, ridging, and tensile failure models, which are simpler and better understood than the complete sea ice deformation model, can be employed. In this case, the sea ice rheology is built up naturally from these elemental failure regimes, depending on sea ice state and stress.

The adopted coulombic failure is based on laboratory experiments (Schulson 2001) and is less mathematically sophisticated than the elastic-decohesive model that not only treats discrete failure but also incorporates brittle and ductile regimes (Schreyer et al. 2006; Sulsky and Paterson 2011). However, there is no evidence to suggest that the elastic-decohesive model describes sea ice rheology better than a simpler, coulombic model. One disadvantage of our model is that coulombic failure is also associated with the corresponding shear along the slip line, although generally speaking the friction coefficient during kinetic sliding is smaller than the internal friction coefficient (Schulson et al. 2006). The situation is similar in the elastic-decohesive model that reduces to a coulombic sliding law after the crack has become open and the decohesive resistance has disappeared. In contrast, the discrete element ice model developed by Hopkins (1996) used for basin-scale calculations (Hopkins et al. 2004; Hopkins and Thorndike 2006) can be easily modified to include different internal and kinetic friction coefficients (Wilchinsky et al. 2010). On the other hand, in this model, crack healing through refreezing is difficult to model unless relaxation to the initial state is adopted for healing (Wilchinsky et al. 2011). The elastic-decohesive model has yet to include crack healing. Our model, however, directly relates the plastic strength of sea ice to its thickness distribution whose evolution is described explicitly and healing occurs through closing and thermodynamic growth.

The coulombic failure mechanism adopted here is similar to that used in numerical modeling by Tremblay and Mysak (1997). The Tremblay and Mysak model, however, takes no account of existing flaws in the determination of new coulombic failure lines. Observations and discrete numerical modeling (Wilchinsky et al. 2011) demonstrate the importance of existing flaws to rheology, which is why we account for them in the present model. Furthermore, the effect of ridging is modeled by Tremblay and Mysak (1997) through a cutoff of the coulombic cone at a constant pressure where the sea ice was assumed to start failing out of plane through ridging. In our model, ridging failure is described through a derived law (Hopkins 1998) and the transition from coulombic to ridging failure occurs naturally, determined by the failure stresses required by these elemental processes. The assumption of discrete failure also allowed us to use the simple, one-dimensional model of ridging perpendicular to the lead as a basic structural element of our sea ice model. This is in contrast to Hibler and Schulson (2000), who assume an ellipticshape attachment to a coulombic cone in order to model the effect of ridging on the sublead scale. Similarly, full isotropic rheologies on the sublead scale were used by Wilchinsky and Feltham $(2004,2006 a)$ in their anisotropic models. This implies a possibility of ridging at any direction in the lead, including along the lead, which is counterintuitive. Depending on the evolution of the sea ice thickness distribution and changes in sea ice stresses, our yield curve changes dynamically.

Our model describes the dominant and complex plastic regime of sea ice failure and should be complemented by the adoption of a subyield rheology. The subyield regime determines how failure and therefore deformation propagates over a sea ice cover. Although a viscous subyield rheology (Hibler 1979; Tremblay and Mysak 1997) is typically used, an elastic subyield rheology will likely ensure a more realistic scaling law of ice deformation (Hopkins and Thorndike 2006; Wilchinsky et al. 2010; Girard et al. 2011).

Here, we present a short discussion of some of the issues involved in implementing our rheology model into a sea ice dynamics model. Such an implementation is beyond the scope of the present work and would involve numerical issues not discussed here. The procedure we describe below for relating stress to strain rate for the given sea ice state (the rheology model) provides a mapping from stress to strain rate. Because it is usual in numerical sea ice dynamics models to determine stress from strain rate (and the sea ice state), the procedure we describe may in this case be implemented iteratively.

In the case of discrete orientational ice categories, the minima of stress invariants necessary to determine failure lines can be found by comparing them for all orientations, which is a straightforward procedure if the stress principal axes are known. In these axes during coulombic failure at a given pressure $p$, the first failure line is determined by finding the orientation where $\tau$ in (4) attains its minimum with the relative lead thickness given by (5). The second failure line is then again determined by where the maximum shear stress $\tau$ attains its minimum, but now it is expressed through (8) given the presence of the couple stress (9). The found $\tau$ is the maximum shear stress determined by the given pressure 

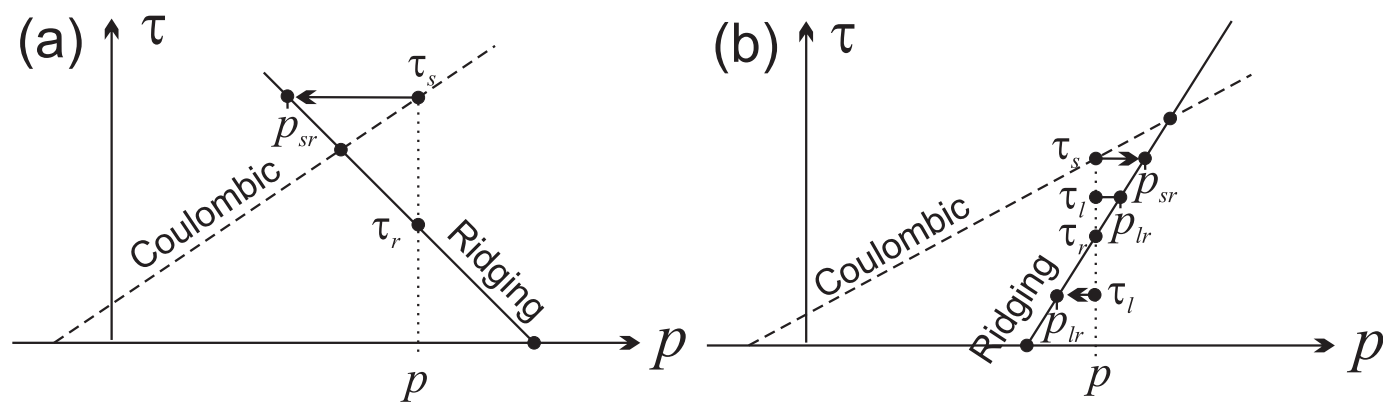

FIG. 8. Two yield curve cases where the coulombic stress lies (a) outside the yield envelope and (b) on the envelope. The ridging branches are produced by ridging of only one lead lying (a) closer to the most compressive axis and (b) closer to the least compressive axis.

$p$ during coulombic failure. The ridging branch of the yield curve is determined by fixing $\tau$ and finding such orientation where the pressure reaches its minimum in (13) or as is explicitly expressed in (30). The involved pressure ridging force $F_{r}$ is given by (12), which employs the participation functions (10) and (11). The tensile failure branch of the yield curve is found similarly to the pressure ridging one: through minimization of the pressure $p$ at a fixed maximum shear stress $\tau$ in (15) that employs the tensile failure force function (14). The found modes of failure determine their corresponding normalized strain rate contributions in the stress principal axes (25) and (28) for coulombic and ridging/ tensile failures to the whole strain rate (29) that involves unknown rates of shear along coulombic lines and closing and opening across ridging and tensile failure lines. The shear, closing, and opening rates (which are, together with the stress principal direction, to be found from the sea ice mass and momentum conservation equations) then determine the ice thickness redistribution rates at failure lines (21) and (22) that enter the ice thickness evolution Eq. (20).

As can be seen from the yield curves-envelopes plotted earlier, the yield curve is determined by various failure modes and it is necessary to determine, for a given pressure $p$, which failure modes define the yield curve. For example, if the ridging branch, represented by only one lead, has a slope of a different sign to the coulombic branch (Fig. 8a), then the found coulombic stresses at a given pressure $p$ will be outside of the failure envelope if, for the chosen pressure, $\tau_{s}$ found from coulombic failure is larger than $\tau_{r}$ found from ridging failure. On the other hand, if the ridging branch has the same sign slope as the coulombic branch (Fig. 8b), then both maximum shear stresses, $\tau_{s}$ and $\tau_{r}$, determined by coulombic and ridging failure, respectively, are possible because they both lie on the combined yield curve.

In a practical application, the yield curve is not known beforehand and so an algorithm for its determination is required. When the whole yield curve is not known, one begins with a prescribed pressure $p$ and determines the maximum shear stress $\tau_{s}$ determined by the coulombic model (Fig. 8). The found $\tau_{s}$ can then be used to find $p_{\mathrm{sr}}$ determined by ridging failure. If $p_{\mathrm{sr}}>p$, then $\left(p, \tau_{s}\right)$ lies on the yield curve; otherwise, coulombic failure does not occur at this pressure; that is, $\left(p, \tau_{s}\right)$ does not lie on the yield curve (e.g., Fig. 8a). At this same pressure $p$, we can consider a lower shear stress $\tau_{l}<\tau_{s}$ : lowering $\tau$ below $\tau_{r}$ can lead to the stress state $\left(p, \tau_{l}\right)$ exiting the yield envelope (e.g., Fig. $8 \mathrm{~b}$ ). If $\tau_{r}$ is not known, then it is again necessary to check if this lower $\tau_{l}$ determines such $p_{\text {lr }}$ found through ridging failure that it is larger than the initially considered pressure $p$ : if $p_{\text {lr }}<p$, then this stress state is outside of the yield envelope and cannot exist and vice versa. Similarly, in order to find a stress for ridging failure, one can begin with a prescribed shear stress $\tau$ and find $p_{r}$ from the ridging model: if the coulombic shear stress $\tau_{\mathrm{rs}}$ corresponding to $p_{r}$ is larger than the prescribed shear stress $\tau$, (i.e., $\tau_{\mathrm{rs}}<\tau$ ), then this ridging branch is on the yield curve; otherwise, it is not.

Apart from sea ice rheology, standard sea dynamics ice models contain two momentum equations (resolved along the chosen coordinate axes) and one mass balance equation. If failure occurs either as coulombic only (which we have taken to be in two leads simultaneously) or ridging-tensile failure in one lead only, then there is only one unknown failure deformation rate, $\dot{\xi}_{s}$ or $\dot{\xi}_{r, o}$. For these failure modes, the prescribed pressure $p$ determines the maximum shear $\tau$ (or vice versa) as well as rate-independent strain rate constituents $\dot{\mathbf{e}}^{s}$ or $\dot{\mathbf{e}}^{r, o}$. Together with the unknown direction of the highest compression $\left(\sigma_{1}\right)$ axis, (e.g., $\theta, p$, and one deformation rate), there are three unknowns that can be determined by the two momentum equations and the mass balance equation.

If combined failure occurs, either as coulombic failure and ridging/tensile failure in one lead simultaneously or as ridging-tensile failure in two leads simultaneously, 
then we have two equations for $p$ and $\tau$, and they are uniquely determined. In this case, there are two unknown lead deformation rates: $\dot{\xi}_{s}$ and $\dot{\xi}_{r, o}$ or $\dot{\xi}_{r, o}^{(1)}$ and $\dot{\xi}_{r, o}^{(2)}$. Together with the most compressive direction $\theta$, there are again three unknowns for the two momentum equations and the mass balance equation.

\section{Concluding remarks}

An anisotropic sea ice model has been proposed that explicitly accounts for coulombic, ridging, and tensile failure. The sea ice state is described by areal thickness distribution functions for isotropic floe ice and orientationdependent lead ice. Ridging of ice in leads and floes redistributes ice thickness in them and transfers ice from leads to floes when ridges become thicker than floes. Opening due to tensile failure and dilatancy during sliding in coulombic failure produces open water and decreases the mean thickness of the leads. The coulombic yield function decreases as lead orientation deviates from the standard critical angles and, due to the presence of cohesion, is larger in thinner leads. Coulombic failure determines formation of new slip lines that are dependent on the orientation and thickness of existing leads. Given discrete orientational ice categories, the minimum of the maximum shear stress that determines the failure line orientation can be found by comparing the maximum shear stress in each category. Similarly, ridging or tensile failure orientations can be found. The direct determination of ridging failure orientations relieves us from adopting an ad hoc extension of the coulombic yield curve for higher pressures (see, e.g., Hibler and Schulson 2000). Depending on the failure regimes, the strain rate consists of the relative contributions from shear and dilatancy along two coulombic slip lines and contraction-extension perpendicular to up to two ridging-tensile failure lines. For typical deformation rates in the Arctic, dilatancy during sliding along coulombic lines results in these lines becoming more prone to ridging failure, which is frequently observed.

The sea ice yield curve is a combination of segments describing the different failure modes (coulombic, ridging, and tensile), merging at points of combined failure modes. The orientation of the segments due to ridging failure depends on the angle of the ridging leads. Considering a scenario of two pairs of leads of different thickness formed at critical angles with regard to different maximum compressive axes, we illustrated the complexity of shapes that a combined yield curve can take. This is due to switching of ridging and coulombic failure between different leads depending on their orientation and thickness at different stresses.
Implementation of our rheology into a numerical sea ice dynamics model will require splitting the usual ice thickness categories into orientation-dependent subcategories in order to model the state of the leads. The floe ice thickness distribution is described by the standard (and separate) isotropic ice thickness categories. The rheology model involves three unknowns including the stress orientation, one or two deformation rates at the failure lines, and one of the stress invariants if there is only one failure mode acting; these three unknowns can be determined using the two momentum and one mass conservation equations. Because the rheology model is written is terms of a relationship where the stress determines the strain rate, an iterative procedure will likely be required for its adoption in standard sea ice models. Although using orientational ice thickness subcategories will require more calculations, because failure occurs only along four directions at most, failure will redistribute the ice thickness only along these directions: the majority of the orientational ice categories will not be affected apart from their consequent renormalization. Similarly, thermodynamic growth affects the same ice thicknesses at all orientations equally and requires only a single calculation. Therefore, the increased level of complexity of the model because of a better representation of sea ice anisotropy should not lead to a significant increase of computational time.

\section{REFERENCES}

Ashby, M. F., and S. D. Hallam, 1986: The failure of brittle solids containing small cracks under compressive stress states. Acta Metall., 34, 497-510, doi:10.1016/0001-6160(86)90086-6.

Bratchie, I., 1984: Rheology of an ice floe field. Ann. Glaciol., 5, 23-28.

Coon, M. D., G. A. Maykut, R. S. Pritchard, D. A. Rothrock, and A. S. Thorndike, 1974: Modelling the pack ice as an elasticplastic material. AIDJEX Bulletin, No. 24, University of Washington, Seattle, WA, 1-105.

—_, G. S. Knoke, D. C. Echert, and R. S. Pritchard, 1998: The architecture of anisotropic elastic-plastic sea ice mechanics constitutive law. J. Geophys. Res., 103 (C10), 21 91521925

- , R. Kwok, G. Levy, M. Pruis, H. Schreyer, and D. Sulsky, 2007: Arctic Ice Dynamics Joint Experiment (AIDJEX) assumptions revisited and found inadequate. J. Geophys. Res., 112, C11S90, doi:10.1029/2005JC003393.

Cowin, S. C., 1974: The theory of polar fluids. Adv. Appl. Mech., 14, 279-347, doi:10.1016/S0065-2156(08)70034-6.

Dempsey, J. P., R. M. Adamson, and S. V. Mulmule, 1999: Scale effects on the in-situ tensile strength and fracture of ice. Part II: First-year sea ice at Resolute, N.W.T. Int. J. Fract., 95 (1-4), 347-366, doi:10.1023/A:1018650303385.

Erlingsson, B., 1988: Two-dimensional deformation patterns in sea ice. J. Glaciol., 34, 301-308.

Feltham, D. L., 2008: Sea ice rheology. Annu. Rev. Fluid Mech., 40, 91-112, doi:10.1146/annurev.fluid.40.111406.102151. 
Girard, L., S. Bouillon, J. Weiss, D. Amitrano, T. Fichefet, and V. Legat, 2011: A new modelling framework for sea-ice mechanics based on elasto-brittle rheology. Ann. Glaciol., 52, 123-132.

Hibler, W. D., III, 1979: A dynamic thermodynamic sea ice model. J. Phys. Oceanogr., 9, 815-846.

— 1980: Modeling a variable thickness sea ice cover. Mon. Wea. Rev., 108, 1943-1973.

— , 2001: Modeling the formation and evolution of oriented fractures in sea ice. Ann. Glaciol., 33, 157-164, doi:10.3189/ 172756401781818680

— , and E. M. Schulson, 2000: On modeling the anisotropic failure and flow of flawed sea ice. J. Geophys. Res., 105 (C7), 17 105-17 120.

Hopkins, M. A., 1996: On the mesoscale interaction of lead ice and floes. J. Geophys. Res., 101 (C8), 18 315-18 326.

_ 1998: Four stages of pressure ridging. J. Geophys. Res., 103 (C10), 21 883-21 891.

— , and A. S. Thorndike, 2006: Floe formation in Arctic sea ice. J. Geophys. Res., 111, C11S23, doi:10.1029/2005JC003352.

_ S. Frankenstein, and A. S. Thorndike, 2004: Formation of an aggregate scale in Arctic sea ice. J. Geophys. Res., 109, C01032, doi:10.1029/2003JC001855.

Jaeger, J. C., and N. G. W. Cook, 1979: Elasticity and strength of rock. Fundamentals of Rock Mechanics, J. C. Jaeger and N. G. W. Cook, Eds., Chapman and Hall, 95-100.

Lewis, J. K., and J. A. Richter-Menge, 1998: Motion-induced stresses in pack ice. J. Geophys. Res., 103 (C10), 21 831-21 843.

Marko, J. R., and R. E. Thomson, 1977: Rectilinear leads and internal motions in the pack ice in the western Arctic Ocean. J. Geophys. Res., 82, 7787-7802.

Overland, J. E., and C. H. Pease, 1988: Modeling ice dynamics of coastal seas. J. Geophys. Res., 93 (C12), 15 619-15 637.

Perovich, D. K., and Coauthors, 1999: Year on ice gives climate insights. Eos, Trans. Amer. Geophys. Union, 80, 485-486.

Pritchard, R. S., 1988: Mathematical characteristics of sea ice dynamics models. J. Geophys. Res., 93 (C12), 15 609-15 618.

Richter-Menge, J. A., and K. F. Jones, 1993: The tensile stress of first-year sea ice. J. Glaciol., 39, 609-618.

— , S. L. McNutt, J. E. Overland, and R. Kwok, 2002: Relating Arctic pack ice stress and deformation under winter conditions. J. Geophys. Res., 107, 8040, doi:10.1029/2000JC000477.

Rothrock, D. A., 1975: The energetics of the plastic deformation of pack ice by ridging. J. Geophys. Res., 80, 4514-4519.

Schreyer, H. L., D. L. Sulsky, L. B. Munday, M. D. Coon, and R. Kwok, 2006: Elastic-decohesive constitutive model for sea ice. J. Geophys. Res., 111, C11S26, doi:10.1029/2005JC003334.

Schulson, E. M., 2001: Brittle failure of ice. Eng. Fract. Mech., 68 (17-18), 1839-1887, doi:10.1016/S0013-7944(01)00037-6.

—, A. L. Fortt, D. Iliescu, and C. E. Renshaw, 2006: On the role of frictional sliding in the compressive fracture of ice and granite: Terminal vs. post-terminal failure. Acta Mater., 54, 3923-3932, doi:10.1016/j.actamat.2006.04.024.

Shen, H. H., W. D. Hibler III, and M. Leppäranta, 1987: The role of floe collision in sea ice rheology. J. Geophys. Res., 92 (C7), 7085-7096.

Smith, R. B., 1983: A note on the constitutive law for sea ice. J. Glaciol., 29, 191-195.

Stern, H. L., and R. W. Lindsay, 2009: Spatial scaling of Arctic sea ice deformation. J. Geophys. Res., 114, C10017, doi:10.1029/ 2009JC005380.

Sulsky, D., and K. Paterson, 2011: Toward a new elastic-decohesive model of Arctic sea ice. Physica D, 240, 1674-1683, doi:10.1016/ j.physd.2011.07.005.

Taylor, P. D., D. L. Feltham, P. R. Sammonds, and D. Hatton, 2006: Continuum sea ice rheology determined from subcontinuum mechanics. J. Geophys. Res., 111, C11015, doi:10.1029/ 2005JC002996.

Thorndike, A. S., D. A. Rothrock, G. A. Maykut, and R. Colony, 1975: The thickness distribution of sea ice. J. Geophys. Res., 80, 4501-4513.

Tremblay, L. B., and L. A. Mysak, 1997: Modeling sea ice as a granular material, including the dilatancy effect. $J$. Phys. Oceanogr., 27, 2342-2360.

Walter, B. A., J. E. Overland, and P. Turet, 1995: A comparison of satellite-derived and aircraft-measured regional surface sensible heat fluxes over the Beaufort Sea. J. Geophys. Res., 100 (C3), 4585-4591

Weiss, J., and E. M. Schulson, 2009: Coulombic faulting from the grain scale to the geophysical scale: lessons from ice.J. Phys. $D$ Appl. Phys., 42, 214017, doi:10.1088/0022-3727/42/21/214017.

$\longrightarrow,-$, and H. L. Stern, 2007: Sea ice rheology from in-situ, satellite and laboratory observations: Fracture and friction. Earth Planet. Sci. Lett., 255 (1-2), 1-8, doi:10.016/j.epsl.2006.11.033.

Wilchinsky, A. V., and D. L. Feltham, 2004: A continuum anisotropic model of sea-ice dynamics. Proc. Roy. Soc. London, 460A, 2105-2140, doi:10.1098/rspa.2004.1282.

_ dynamics. J. Mech. Phys. Solids, 54, 1147-1185, doi:10.1016/ j.jmps.2005.12.006

_ and - 2006b: Modelling the rheology of sea ice as a collection of diamond-shaped floes. J. Non-Newtonian Fluid Mech., 138, 22-32, doi:10.1016/j.jnnfm.2006.05.001. , and —- 2011: Modeling Coulombic failure of sea ice with leads. J. Geophys. Res., 116, C08040, doi:10.1029/ 2011JC007071.

- _ — , and M. A. Hopkins, 2010: The effect of shear rupture on aggregate scale formation in sea ice. J. Geophys. Res., 115, C10002, doi:10.1029/2009JC006043.

$-\ldots, \ldots$, and ——, 2011: Modelling the reorientation of seaice faults as the wind changes direction. Ann. Glaciol., 52, 83-90. 